\title{
Cortical Gyrification Induced by Fibroblast Growth Factor 2 in the Mouse Brain
}

\author{
Brian G. Rash, ${ }^{1 *}$ Simone Tomasi, ${ }^{1 *}$ H. David Lim, ${ }^{1}$ Carol Y. Suh, ${ }^{1}$ and Flora M. Vaccarino ${ }^{1,2,3}$ \\ ${ }^{1}$ Child Study Center, ${ }^{2}$ Department of Neurobiology, and ${ }^{3}$ Kavli Institute for Neuroscience, Yale University, New Haven, Connecticut 06520
}

Gyrification allows an expanded cortex with greater functionality to fit into a smaller cranium. However, the mechanisms of gyrus formation have been elusive. We show that ventricular injection of FGF2 protein at embryonic day 11.5- before neurogenesis and before the formation of intrahemispheric axonal connections-altered the overall size and shape of the cortex and induced the formation of prominent, bilateral gyri and sulci in the rostrolateral neocortex. We show increased tangential growth of the rostral ventricular zone (VZ) but decreased Wnt3a and Lef1 expression in the cortical hem and adjacent hippocampal promordium and consequent impaired growth of the caudal cortical primordium, including the hippocampus. At the same time, we observed ectopic Er81 expression, increased proliferation of Tbr2-expressing $\left(\mathrm{Tbr} 2^{+}\right.$) intermediate neuronal progenitors (INPs), and elevated $\mathrm{Tbr}^{+}{ }^{+}$neurogenesis in the regions that undergo gyrification, indicating region-specific actions of FGF2 on the VZ and subventricular zone (SVZ). However, the relative number of basal radial glia - recently proposed to be important in gyrification-appeared to be unchanged. These findings are consistent with the hypothesis that increased radial unit production together with rapid SVZ growth and heightened localized neurogenesis can cause cortical gyrification in lissencephalic species. These data also suggest that the position of cortical gyri can be molecularly specified in mice. In contrast, a different ligand, FGF8b, elicited surface area expansion throughout the cortical primordium but no gyrification. Our findings demonstrate that individual members of the diverse Fofgene family differentially regulate global as well as regional cortical growth rates while maintaining cortical layer structure.

\section{Introduction}

Total surface area of the cerebral cortex is one major variable in mammalian species, but so too is the size and shape of different lobes or regions. For instance, compared with other cortical areas, the frontal lobe is expanded many fold in primates (Rakic, 2009). In contrast, the hippocampus constitutes a much larger proportion of the rodent cortex than in primates. Thus, the cortex expands in a region-specific manner in higher mammals, yet the mechanisms regulating the growth of specific cortical regions are not understood.

The cerebral cortex is derived from an embryonic layer of stem cells, known as radial glial (RG) cells, that diversifies into ventricular zone (VZ) and subventricular zone (SVZ) (Malatesta et al., 2000; Kriegstein et al., 2006; Dehay and Kennedy, 2007; Rakic, 2009). During embryonic day 10 (E10) to E12 in the mouse and weeks 5-10 of gestation in humans, there is rapid expansion of the stem cell pool (Rakic, 1988, 1995). The time of onset of neurogenesis, normally at approximately E11.5 in mice (Takahashi et al., 1996, 1999), and the

\footnotetext{
Received July 29, 2012; revised May 4, 2013; accepted May 15, 2013

Author contributions: B.G.R., S.T., and F.M.V. designed research; B.G.R., S.T., H.D.L., and C.Y.S. performed research; B.G.R., S.T., and F.M.V. analyzed data; B.G.R., S.T., and F.M.V. wrote the paper.

This work was supported by National Institutes of Health Grant R01 MH067715 (F.M.V) and T32 MH18268 fellowship (B.G.R) and a Brown-Coxe postdoctoral fellowship (B.G.R.). We thank Allyson Vermaak for excellent surgical assistance and Drs. Nenad Sestan, Karen Smith, and Hanna Stevens for helpful discussions.

${ }^{*}$ B.G.R. and S.T. contributed equally to this work.

The authors declare no competing financial interests.

Correspondence should be addressed to Flora M. Vaccarino, Yale Child Study Center, Yale University School of Medicine, P.0. Box 207900, New Haven, CT 06520. E-mail: flora.vaccarino@yale.edu.

DOI:10.1523/JNEUROSCI.3621-12.2013

Copyright $\odot 2013$ the authors $\quad 0270-6474 / 13 / 3310802-13 \$ 15.00 / 0$
}

rate of expansion of the RG stem cell pool are regulated by fibroblast growth factor (FGF) and Notch signaling (Vaccarino et al., 1999; Raballo et al., 2000; Ohtsuka et al., 2001; Stevens et al., 2010; Rash et al., 2011). The rate of stem cell depletion via neurogenesis is key to the expansion of cortical surface area. However, whether this expansion is uniform or differentially regulated across regions of the pallium has not been established.

In gyrencephalic species, bends and folds in the neuroepithelium appear early in neurogenesis and mature over a very long period of development, leading to the primarily conserved spatial pattern of the major sulci and gyri. Four very different mechanisms for gyrus formation have been proposed in recent years. Rapid expansion of the cortical neuroepithelium is thought to elicit gyrencephaly by making it more flexible and prone to folding during brain growth in a limited volume (Rakic, 1988, 2009). Alternatively, axonal fibers have been proposed to pull cortical regions together by mechanical tension (Van Essen, 1997; Nie et al., 2012). Another group observed that excess differentiation of SVZ progenitors regionally coincides with gyrus formation in primate cortical development (Kriegstein et al., 2006). Recently, the "basal" or "outer" radial glial (oRG) cells, which are more abundant in ferrets and primates, have been postulated to drive the bending and folding of the cortical wall during gyrification (Lui et al., 2011; Reillo et al., 2011; Wang et al., 2011). Most of these studies are comparative cross-species analyses that have not experimentally induced gyrification in a lissencephalic mammal to study the origins of a gyrencephalic cortex.

We show that the cortical primordium responds very differently to two ligands of the FGF family, FGF2 and FGF8b. Notably, 
Table 1. Primary antibodies used in the experiments

\begin{tabular}{llll}
\hline Antibody & Species & Source & Dilution \\
\hline Tbr1 & $\mathrm{Rb}$ & Millipore & $1: 1000$ \\
Tbr2 & $\mathrm{Rb}$ & Abcam & $1: 1000$ \\
Tbr2 & $\mathrm{Ck}$ & Millipore & $1: 500$ \\
PH3 & $\mathrm{Rt}$ & Sigma & $1: 1000$ \\
Pax6 & $\mathrm{Rb}$ & Millipore & $1: 1000$ \\
BIII Tubulin & $\mathrm{Ms}$ & Promega & $1: 2000$ \\
Ki67 & $\mathrm{Rb}$ & Vector Laboratories & $1: 1000$ \\
BrdU & $\mathrm{Rt}$ & Accurate Chemical and Scientific Corp. & $1: 400$ \\
GFP & $\mathrm{Ck}$ & Abcam & $1: 1000$ \\
Ctip2 & $\mathrm{Rt}$ & Abcam & $1: 1000$ \\
Cux1 & $\mathrm{Rb}$ & Santa Cruz Biotechnology & $1: 100$ \\
ZFPM2 & $\mathrm{Gt}$ & Santa Cruz Biotechnology & $1: 250$ \\
Smi32 & $\mathrm{Ms}$ & Covance & $1: 500$ \\
\hline
\end{tabular}

Rb, Rabbit; Ck, chick; Ms, mouse; Rt, rat; Gt, goat.

using intraventricular injections of FGF2 protein at E11.5, we have created a novel mouse model of cortical gyrification and explored the mechanism of gyrencephaly. To our knowledge, this is the first mouse model of fully layered neocortical gyri/sulci that survives healthily until adulthood.

\section{Materials and Methods}

Animals, in utero FGF protein injection, and BrdU labeling. All experimental procedures involving animals were performed in accordance with the policies of the Yale Institutional Care and Use Committee. Timed pregnant CD-1 mice were obtained from Charles River Laboratories and housed at the Yale Sterling Hall of Medicine animal facility. Pregnant dams were anesthetized by isoflurane, and embryos were injected with either human recombinant FGF2 (hrFGF2; Invitrogen) or hrFGF8b (R\&D Systems) or vehicle alone at E11.5 and allowed to develop in utero for $8 \mathrm{~h}$ or until E13.5 or E18.5. Some animals were allowed to give birth, and the injected pups were analyzed at adulthood. A single intraperitoneal injection of BrdU (Sigma) was administered at $50 \mathrm{mg} / \mathrm{kg}$ to pregnant dams $4 \mathrm{~h}$ after in utero FGF2 injection to label dividing cells that were responding to excess FGF protein. Of 77 total animals analyzed in this study, 56 were used for FGF2 injections or controls, eight for FGF8b experiments, and 13 for vehicle-control experiments. For FGF2 injections, 21 of 28 FGF2-injected embryos, which were identified during surgery as having a high probability of success, were highly phenotypic and showed clear evidence of cortical gyrus/sulcus formation at the age of analysis.

Tissue preparation. Embryonic and adult brains were dissected at defined stages and immediately placed in DEPC-treated PBS and fixed in $4 \%$ PFA overnight at $4^{\circ} \mathrm{C}$. On the following day, brains were equilibrated overnight in 25\% sucrose/DEPC-PBS and frozen in OCT. Sections (25 $\mu \mathrm{m})$ were produced using a Leica cryostat, stored at $-80^{\circ} \mathrm{C}$, and then processed for immunohistochemistry, BrdU labeling, or RNA in situ hybridization.

Immunohistochemistry and in situ hybridization. Immunohistochemistry was performed as previously described (Smith et al., 2006). Primary antibodies were detected using Alexa-Fluor-conjugated secondary antibodies from (Invitrogen) or DyLight secondary antibodies from Jackson ImmunoResearch. Primary antibodies and dilutions are shown in Table 1. The immunostaining for the Tbr1 and Pax6 used an antigen retrieval step by incubating for $5 \mathrm{~min}$ in $0.01 \mathrm{M} \mathrm{Na}$ citrate, $\mathrm{pH} 6.0$, at $95^{\circ} \mathrm{C}$. Detection of BrdU was performed as described previously (Raballo et al., 2000). In situ hybridization was performed using alkaline phosphatase detection of digoxigenin-labeled riboprobes as described previously (Grove et al., 1998; Rash and Grove, 2007). Fluorescence threedimensional (3D) optical sectioning and bright-field imaging used a Carl Zeiss Apotome or Axioplan microscope and Axiovision 4.8 software.

$R N A$ isolation, cDNA synthesis, and qRT-PCR analysis. To quantify Lef1 expression in the embryonic hippocampal formation, mRNA was isolated from E13.5 embryos ( $n=6$ for vehicle-injected and $n=3$ for FGF2-injected group) from Formalin-fixed coronal sections mounted onto slides. The dentate gyrus was carefully dissected from the hippocampal primordium, and mRNA was extracted using the RecoverAll kit (Ambion) following the instructions of the manufacturer for nucleic acid isolation from Formalin-fixed samples and then treated with DNase I to remove any trace amounts of DNA. RNA yield and integrity was assessed using the Agilent 2100 bioanalyzer nanochip and an RNA LabOn-A-Chip kit. cDNA was synthesized using the SuperScript III FirstStrand Synthesis System for qRT-PCR (Invitrogen). Quantitative RT-PCR was performed on a StepOne Real Time PCR System (Applied Biosystems) using TaqMan probes for actin and Lef1. All reactions were normalized to actin and presented as relative expression changes compared with control.

Adenovirus-GFP labeling of cortical progenitors. Adenovirus-pCMVGFP was purchased from Addgene and injected (titer, $1.0 \times 10^{10} \mathrm{pfu} / \mathrm{ml}$ ) alone or together with $100 \mathrm{ng}$ of hrFGF2 at E11.5. Brains were collected at E13.5, processed, and immunostained for eGFP, Pax6, and Tbr2. eGFPlabeled cells from virus-injected $(n=162)$ and virus-plus-FGF2-injected ( $n=402)$ embryos were imaged using $40 \times Z$-stacks of optical slices using a Carl Zeiss Apotome running Axiovision 4.8. Images were rotated in three dimensions, and GFP-positive $\left(\mathrm{GFP}^{+}\right)$cell morphology was examined for the presence of an apical process extending toward the lateral ventricles, as well as Pax6 and Tbr2 expression.

Morphometric analysis, densitometry, and cell population quantification. Morphometric and cell population estimates were performed using a Carl Zeiss Axioskop 2 Mot Plus microscope operating Stereoinvestigator and Neurolucida systems (MicroBrightField). To obtain cortical cell counts and cortical volumes, the rostrocaudal axes of cortical hemispheres were sampled in their entirety using regular section intervals, and nuclear profiles of cell populations were randomly sampled using the optical fractionator probe. Stereological estimates of Tbr1/BrdU, total Tbr1 number, and Tbr1 density measurements were performed using Stereoinvestigator in the rostral cortical primordium (rostral to the hippocampal primordium, not including the olfactory bulbs), sampling every 10th section. Cortical and VZ surface areas were estimated by tracing contours of cortical marginal zone or ventricular surface in Neurolucida for every 10th section and computing a product of average contour length, average measured section thickness, and the total number of cortical sections. The estimates are likely a slight underestimate of total surface area because the first and last sections included in the analysis are the first and last sections to show a ventricular cross-section, and thus the extreme cortical poles are excluded. Surface area measurements of vehicle-only-injected controls were compared with their respective littermates, and no differences were detected. Because vehicle-injected controls did not differ in surface area from littermates, we used non-injected littermates for the comparison of surface area between FGF2- and FGF8injected embryos. Total cortical surface area included the neocortical and hippocampal primordia, bounded by the piriform cortex (laterally) and the corticoseptal boundary, cortical hem (medially).

Area densitometry for BrdU signal was performed to detect the total amount of labeling. At $48 \mathrm{~h}$ after BrdU injection, this gives a more accurate indication of the amount of BrdU incorporation than counting BrdU immunolabeled cells; because of excessive dilution of the label during repeated cell divisions, most cells in the VZ, for instance, show only a few puncta. Total BrdU densitometry was performed in $12 \mu \mathrm{m}$ $Z$-stacks in NIH ImageJ. The VZ was divided from the remainder of the cortical wall and the total BrdU signal measured. The ratio of non-VZ over VZ corresponds with the amount of BrdU that remained in the VZ or exited to the SVZ or cortical plate.

For $3 \mathrm{D}$ reconstruction, the whole-brain surface profile was traced in cresyl violet-stained serial sections at $2.5 \times$ magnification using Neurolucida (version 9). Every fifth section was traced, superimposed to the previous one, and aligned on the basis of well-defined anatomical landmarks, such as the ventricles or corpus callosum. Traced contours were then $3 \mathrm{D}$ rendered using the built-in solid modeling algorithm.

Statistical analyses. To compute significance levels for most parameters measured, one-way or two-way ANOVA followed by Bonferroni's post hoc test was performed using Statview 4.0 or an online $t$ test calculator (http://studentsttest.com/); $\chi^{2}$ statistic was adopted to compare the fractions of double-positive cells after BrdU labeling and the proportion of 
oRG candidates after adenovirus-GFP labeling of cortical progenitors. Error bars represent SE, and a significance threshold of $p<0.05$ was used.

\section{Results}

Normally, the early telencephalon expresses several FGF ligands. At approximately E10-E12, the rostral midline region expresses FGF8, FGF17, and FGF18, whereas the ventral telencephalon expresses FGF15 (Rash and Grove, 2006; Cholfin and Rubenstein, 2007; Hébert and Fishell, 2008). FGF9, FGF10, and FGF2 are expressed more broadly in the dorsal telencephalon (Vaccarino et al., 1999; Raballo et al., 2000; Sahara and O'Leary, 2009; Seuntjens et al., 2009). FGF2 has been shown previously to increase the total number of neurons in the mouse cerebral cortex and to promote self-renewal of cortical progenitor cells (Vaccarino et al., 1999; Raballo et al., 2000). FGF2 binds to all three FGF receptors (FGFRs) expressed in the cortical primordium (Chellaiah et al., 1999), which together promote RG self-renewal (Rash et al., 2011), suggesting that FGF2 might therefore have a strong enough capacity to substantially alter the growing cortical surface area, if administered early enough in cortical development. In contrast, FGF8b binds primarily to FGFR3 (Chellaiah et al., 1999) but has been shown to act principally as a morphogen (FukuchiShimogori and Grove, 2001; Toyoda et al., 2010), although it has been also shown to promote growth of the cortical primordium (Storm et al., 2006). Therefore, we chose to test the capacity of these two diverse FGF ligands in regulating cortical growth and morphogenesis during the expansion phase of cortical development (E11.5).

\section{Functional diversity and regional} specificity of individual FGF ligands in the regulation of neocortical growth A single injection of FGF2, delivered to the telencephalic ventricles during the RG expansion phase at E11.5, resulted in substantially altered cortical surface area by E13.5, with a major change in forebrain shape. Although the rostral cortical primordium was increased in surface area in FGF2-injected embryos, the caudal cortical primordium was reduced in surface area compared with vehicle-injected controls or age-matched control littermates (Fig. $1 A, B, D, E, G$ ), suggesting that FGF2 is acting in opposite ways on rostral versus caudal cortical growth. Inward bulges protruding into the ventricle were ob-

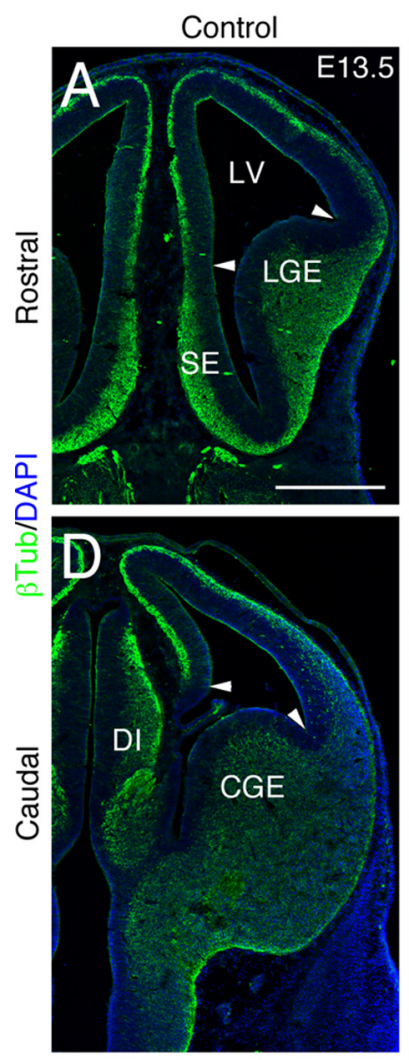

100ng FGF2 @ E11.5
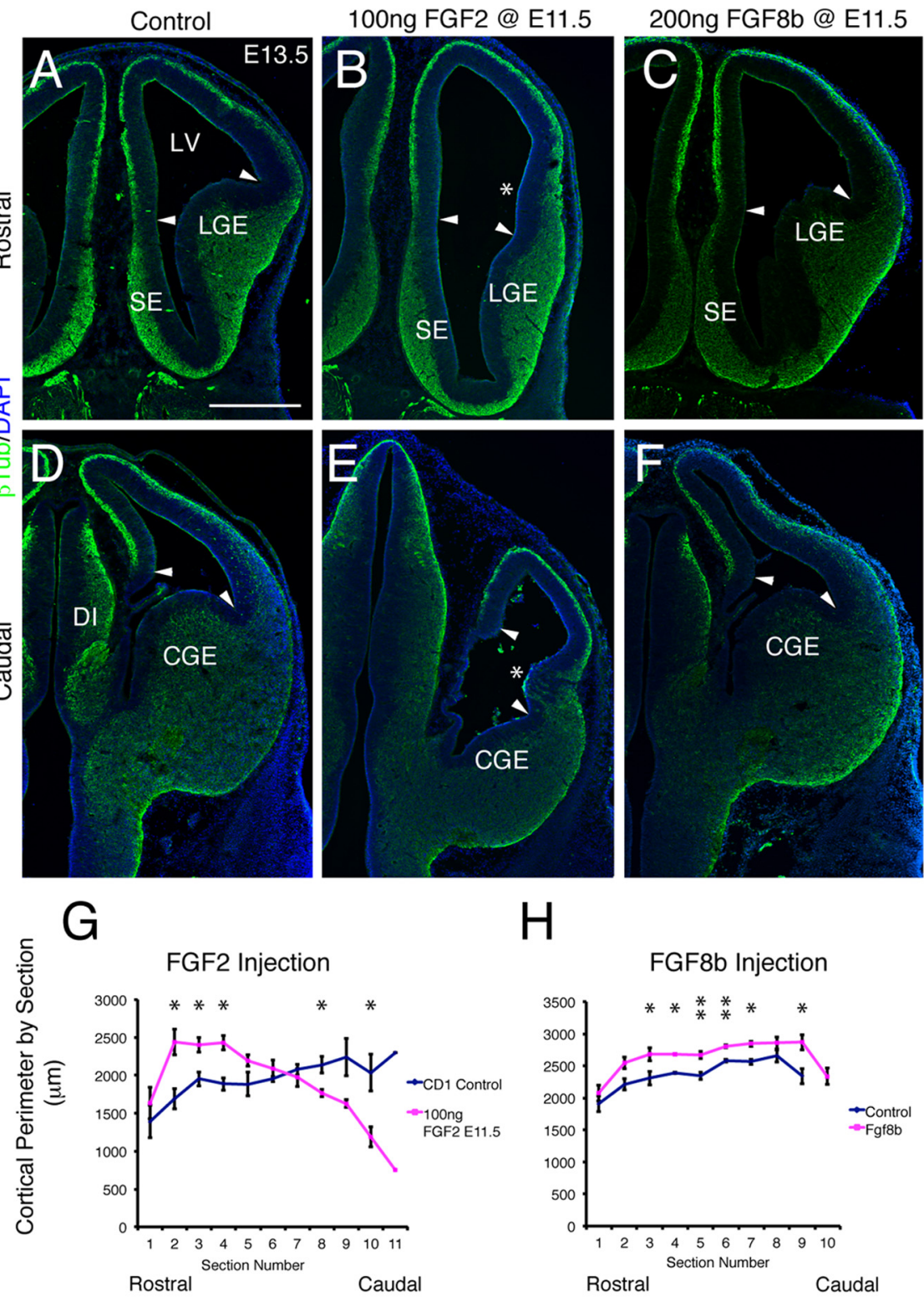

$\mathrm{H}$
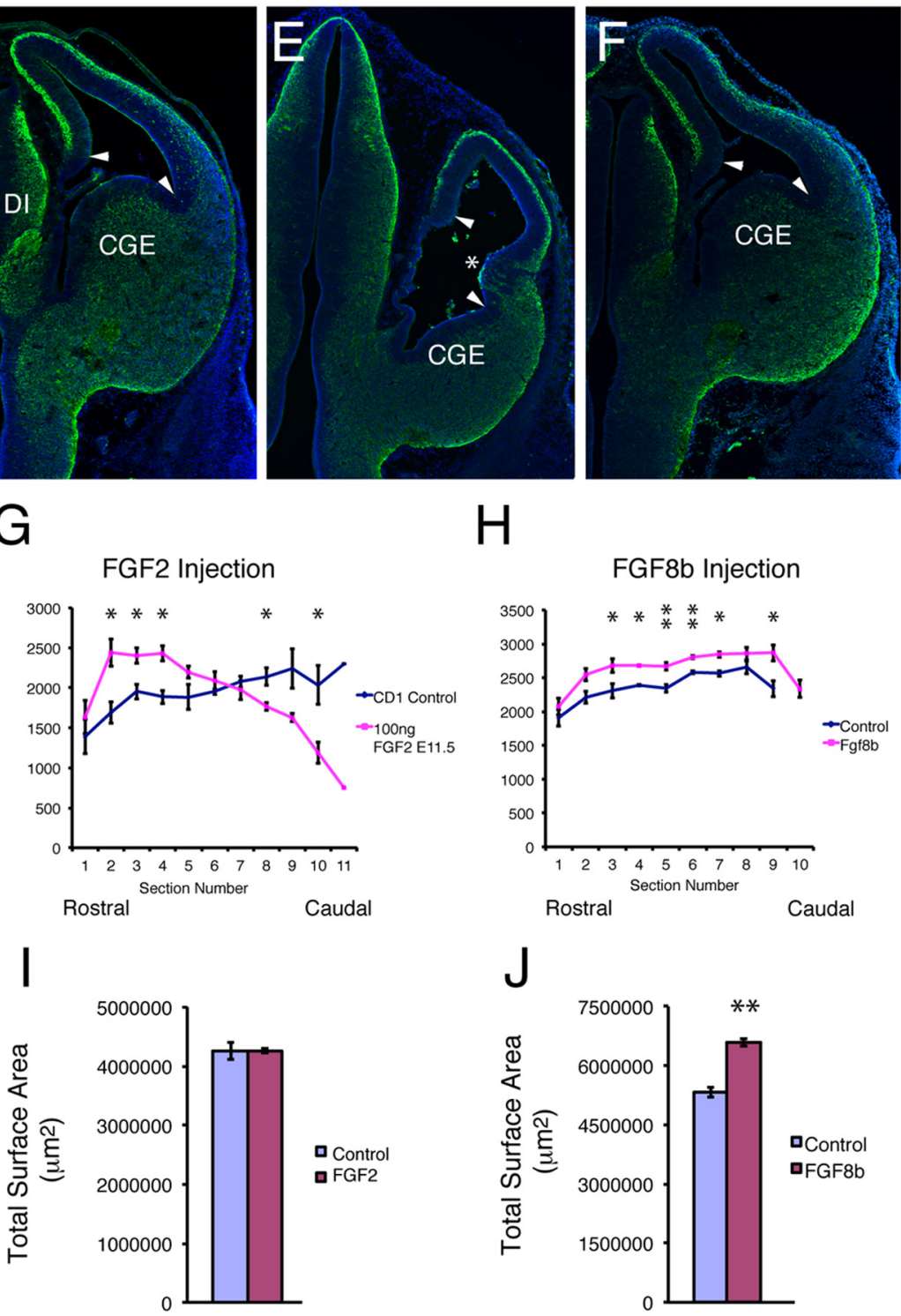

Figure 1. Intraventricularinjection of FGF2 or FGF8b atE11.5 produces different effects on cortical surface expansion by E13.5. Embry0s injected with FGF2 $(\boldsymbol{B}, \boldsymbol{E})$, showed different rostral and caudal effects compared with control littermates $(\boldsymbol{A}, \boldsymbol{D})$. Rostrally, the lateral ventricles were expanded and the perimeter of the cortical VZ (area between arrowheads) was substantially larger than controls $(\boldsymbol{A}, \boldsymbol{B}, \boldsymbol{G})$. In contrast, the caudal cortical primordium appeared shrunken, but the diencephalon expanded, compared with control littermates or vehicle-injected controls $(\boldsymbol{D}, \boldsymbol{E}, \boldsymbol{G})$. The rostral perimeter increase was compensated for by decreased caudal perimeter, and the total surface area of the FGF2-injected cortex was not significantly different from controls $(I ; n=3$ controls and $n=3$ for 100 ng FGF2 embryos; $p=$ 0.95). In contrast, embryos injected with FGF8b showed modest increases in cortical perimeter both rostrally and caudally, and the brain was substantially longer along the rostrocaudal axis $(\boldsymbol{C}, \boldsymbol{F}, \boldsymbol{H})$. Overall, cortical surface area was significantly increased after FGF8b injection by $23.7 \%$ ( $J ; n=3$ controls and $n=3$ for $200 \mathrm{ng}$ FGF8b-injected embryos; $p=0.002$ ). LV, Lateral ventricle; (GE, caudal ganglionic eminence; SE, septum; DI, diencephalon. Scale bar, $1 \mathrm{~mm} .{ }^{*} p<0.05 ;{ }^{* *} p<0.005$. 


\section{E13.5 +100ng FGF2}

Control @ E11.5
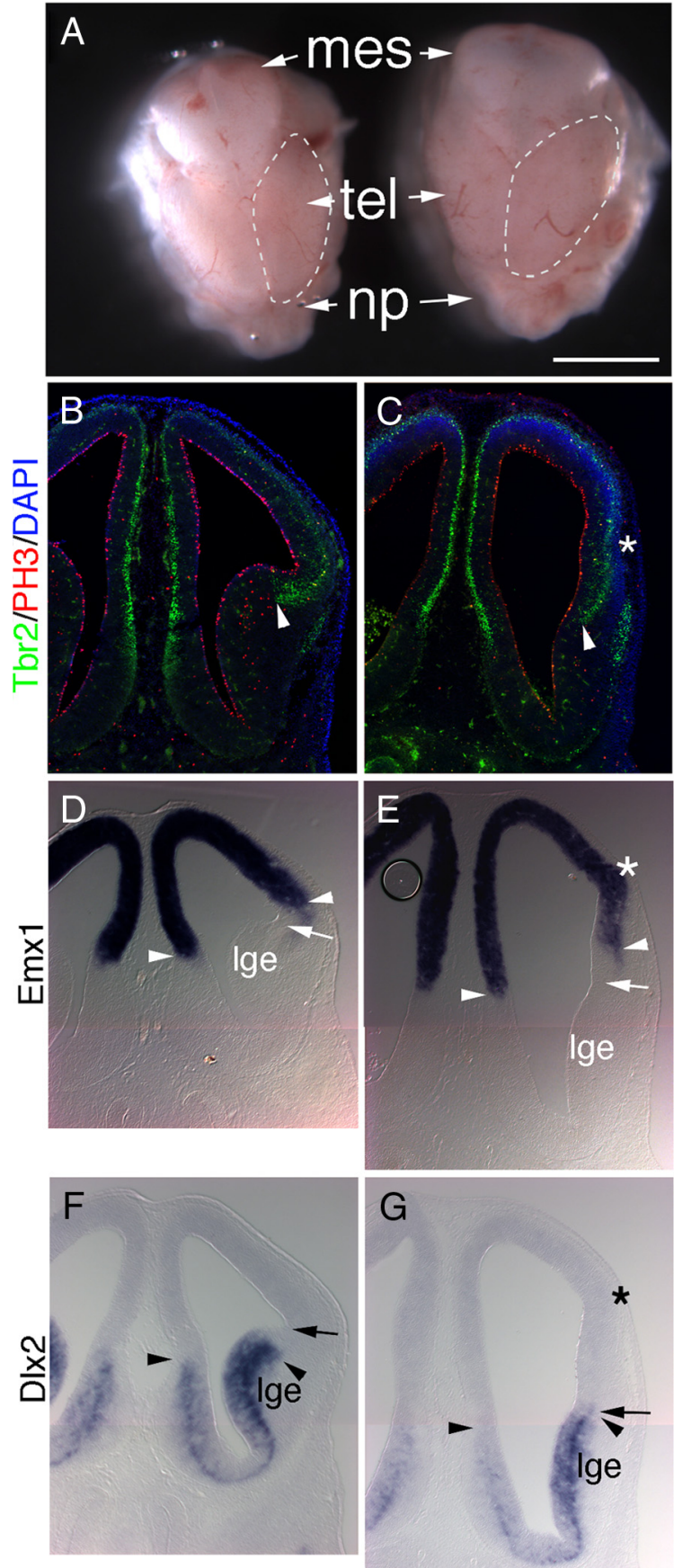

\section{G}

$x$

\section{H DIx2 surface area (anterior vs posterior)}

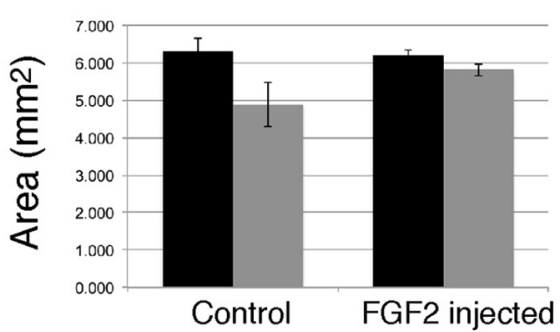

Figure 2. Intraventricular injection of FGF2 induces tangential expansion of rostral cortical VZ and early folding of the Pax6 ${ }^{+} / \mathrm{Emx}{ }^{+}$cortical wall by E13.5. After FGF2 injection at E11.5, embryonic brains are significantly larger than controls $(\boldsymbol{A})$. Immunohistochemistry for Tbr2/PH3 served both rostrally and caudally, although the degree of inward bowing varied between embryos, presumably depending on the precise embryonic age (which normally varies several hours as conception occurs) and dosage of FGF2 received. Total cortical surface area was not modified by FGF2 injection as a result of these differential effects (Fig. $1 I$ ). Vehicle injection (PBS $+0.1 \%$ BSA) showed no difference in total surface area and no rostrocaudal differences were detected $\left(4.35 \pm 0.210 \times 10^{6} \mu \mathrm{m}^{2}\right.$ for controls and $4.19 \pm 0.147 \times 10^{6} \mu \mathrm{m}^{2}$ for vehicle-injected controls $(n=7$ and $n=6$, respectively; $p=0.55)$.

We then asked whether the ability of FGF2 to induce morphogenetic effects was shared with another major telencephalic FGF ligand, FGF8b. We injected FGF8b into the telencephalic ventricles at E11.5, like FGF2, and collected the embryos at E13.5. We found a different pattern of effects; this time, we saw an equal albeit more modest expansion of the cortical domain both rostrally and caudally, with an elongation of the rostrocaudal length of the cortical primordium (Fig. $1 A, C, D, F, H, J, p=0.002$ ). This result supports the hypothesis that different FGF ligands contribute in different ways to region-specific growth of the cerebral cortex.

Staining for $\beta$ III-tubulin showed a preplate that was thicker laterally than medially, indicating that the normal lateral-to-medial gradient of neurogenesis was intact (Fig. $1 B$ ), but the cortical primordium consistently showed buckling associated with increased surface area in rostrolateral regions (Figs. $1 B, 2 C, E, G$, asterisks) as well as sometimes caudally (Fig. $1 E$, asterisk). Occasional heterotopic $\beta$ III tubulin ${ }^{+}$neurons were observed caudally in the VZ at this age (Fig. $1 E$ ) but not rostrally or in subsequent development. To determine which telencephalic regions were most affected by growth or patterning effects, we first determined the position of the corticostriatal and corticoseptal boundaries by staining for Emxl and Tbr2 - markers for the developing cortical domain — together with Dlx 2 for the ventral telencephalon. We found a large increase in rostral ventricle size together with increased rostral cortical surface area (Fig. $2 B-G$ ). The overall surface area for $D l \times 2^{+}$ventral telencephalon was not significantly different from controls, suggesting that FGF2 has a more pronounced role in promoting cortical expansion, but ventral telencephalic volume appeared quite reduced (Fig. $2 \mathrm{~F}-\mathrm{H})$. We also observed slightly altered dorsoventral patterning, because the dorsal boundary of the $D l \times 2$ domain (Fig. $2 F$, G, black arrowheads) was slightly shifted dorsally relative to the morphological pallial-subpallial boundary (Fig. $2 F, G$, black arrows).

\section{Decreased Wnt $3 a$ expression in the cortical hem and excess} FGF pathway activation in the rostrolateral SVZ

To confirm increased FGF activity attributable to the injection, we performed in situ hybridization for the downstream FGF pathway genes Spry1 and Er81 (activated by FGF activity) and CoupTF1 (repressed by FGF activity). Just $8 \mathrm{~h}$ after injection, we found massively increased Spry1 expression and substantially

\footnotetext{
$\leftarrow$

$(\boldsymbol{B}, \boldsymbol{C})$ indicates the extent of the cortical domain (SVZ) and normal location of ventricular and ab-ventricular mitoses. The $E m \times 1$ domain, often considered the best neocortical marker, is shown by in situ hybridization (D, E; boundaries shown by arrowheads). Note that the Emx1 domain does not fully extend to the pallial-subpallial boundary in both control and FGF2injected embryos (arrows). In FGF2-injected embryos, the D/x2 domain ( $\boldsymbol{F}, \boldsymbol{G}$, arrowheads) appeared to extend farther toward the morphological pallial-subpallial boundary (arrows). Total surface area of the D/x2 domain in the rostral (black bars) and caudal (gray bars) telencephalon did not show significant differences $(\boldsymbol{H})$. In the dorsal cortical domain, we consistently observed buckling of the cortical wall (asterisks in $\boldsymbol{C}, \boldsymbol{E}, \boldsymbol{G}$ ). mes, mesencephalon; tel, telencephalon; np, nasal process. Scale bar: $\boldsymbol{A}, 1.8 \mathrm{~mm} ; \boldsymbol{B}-\boldsymbol{G}, 1 \mathrm{~mm}$.
} 


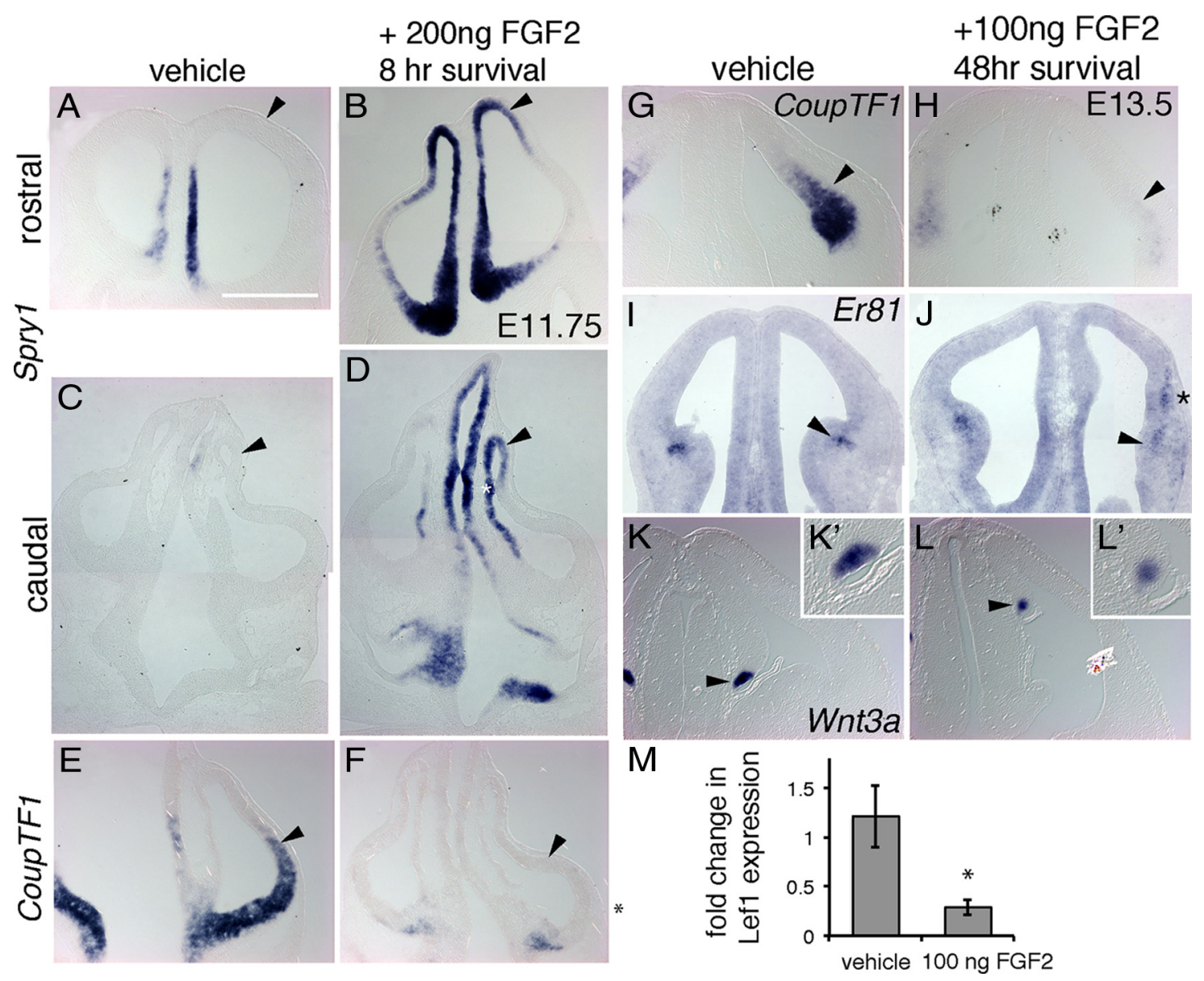

Figure 3. FGF2 injection alters canonical FGF-responsive gene expression within $8 \mathrm{~h}$, downregulates Wnt $3 a$ in the cortical hem, and induces ectopic Er81 within the folding cortical region. Excess transcription of Spry $1 \mathrm{mRNA}$ was detected in both the rostral and caudal cortex $8 \mathrm{~h}$ after FGF2 injection $(\boldsymbol{A}-\boldsymbol{D})$. In contrast, CoupTF1 mRNA was severely downregulated $(\boldsymbol{E}, \boldsymbol{F})$ in the same time period. Downregulation of CoupTF1 mRNA persisted $48 \mathrm{~h}$ after FGF2 injection $(\boldsymbol{G}, \boldsymbol{H})$. Ectopic Er81 mRNA expression was found in the rostrolateral cortical primordium, within the SVZ region, coinciding with early cortical folding $(I, J$, asterisk; arrowheads in $I, J$ indicate pallial-subpallial boundary). In this embryo, folding was observed unilaterally, coinciding with Er81 actopic expression. Wnt3a in the cortical hem was substantially reduced in E13.5 FGF2-injected embryos injected at E11.5 $\left(\boldsymbol{K}, \boldsymbol{K}^{\prime}, \boldsymbol{L}, \boldsymbol{L}^{\prime}\right) . \boldsymbol{M}$, Lef1 mRNA was significantly decreased in the caudomedial pallium, comprising the anlage of the hippocampus, by qRT-PCR. $n=6$ controls and $n=3$ FGF2 injected. Scale bar: $I, J, 1.2 \mathrm{~mm}$; all other panels, $1 \mathrm{~mm} .{ }^{*} p=0.02$.

decreased expression of CoupTF1 (Fig. 3A-F, arrowheads), indicating that the FGF2 injection quickly hyperactivated the canonical downstream FGFR pathway in the cortical primordium as well as the diencephalon. Note the increase in Spry1 expression in the caudomedial telencephalon corresponding with the hippocampal primordium (Fig. 3D, white asterisk). In situ hybridization for CoupTF1 at E13.5 revealed persistent, severe downregulation $48 \mathrm{~h}$ after FGF2 injection (Fig. 3G,H, arrowheads).

Er81 was also expressed near the edge of the lateral ganglionic eminence (LGE) (Fig. 3I, arrowhead), and although this domain was not expanded in FGF2-injected embryos, Er81 was found to be ectopically expressed dorsally, in the apparent SVZ region of the buckling lateral cortex ( $n=3$; Fig. 3J, asterisk), indicating that ectopic FGF activity may have localized effects on cortical development that regionally coincide with cortical folding. Remarkably, we saw Er81 ectopic expression in this region only when cortical surface enlargement and folding of the wall was noticed, as in Fig. 3J, in which a unilateral effect was observed. Because we observed decreased hippocampal size after FGF2 injection (Fig. 1) and because growth of the hippocampus is known to depend on the mitotic activities of Wnt expression in the cortical hem (Grove et al., 1998; Lee et al., 2000), we assessed Wnt3a mRNA expression. Indeed, Wnt3a in the cortical hem appeared substantially downregulated in all embryos examined $(n=3$ controls; $n=3$ FGF2-injected; Fig. $\left.3 K, K^{\prime}, L, L^{\prime}\right)$. To demonstrate globally decreased activation of canonical Wnt signaling in the nearby dentate gyrus, we assessed levels of Lef1, a gene activated downstream of the classic Wnt- $\beta$-catenin pathway, by qRTPCR. Indeed, Lef 1 mRNA is decreased in the caudomedial cortex after FGF2 injections (Fig. $3 M, p=0.02$ ).

\section{Locally increased neurogenesis in the rostrolateral cortex of FGF2-injected embryos}

The total number of Tbr ${ }^{+}$cells in the rostral cortex was not statistically different between the groups (control, 46,966 \pm 5744; FGF2, 58,296 $\pm 21,680 ; p=0.6397)$. Tbr1 density was also not statistically different $(0.00027 \pm 0.00006$ vs $0.0003 \pm 0.00005$ cells $/ \mu \mathrm{m}^{3}$ in control vs FGF2 injected, respectively). However, we observed a change in the time course of neurogenesis. To assess neurogenesis directly, we administered a pulse of BrdU to pregnant dams carrying embryos injected with FGF2 at E11.5. BrdU was given $4 \mathrm{~h}$ after FGF2 injection to label cortical progenitors that were responding in the short term to FGF2 signaling. Embryos collected $48 \mathrm{~h}$ after FGF2 injection (E13.5) were sectioned and double stained for $\mathrm{BrdU}$ and Tbr1 to determine the fraction of neurons generated around the time of the BrdU injection (Fig. $4 A-H)$. Stereological examination of the whole rostral cortex indicated that the average proportion of $\mathrm{Tbr} 1^{+}$neurons labeled 

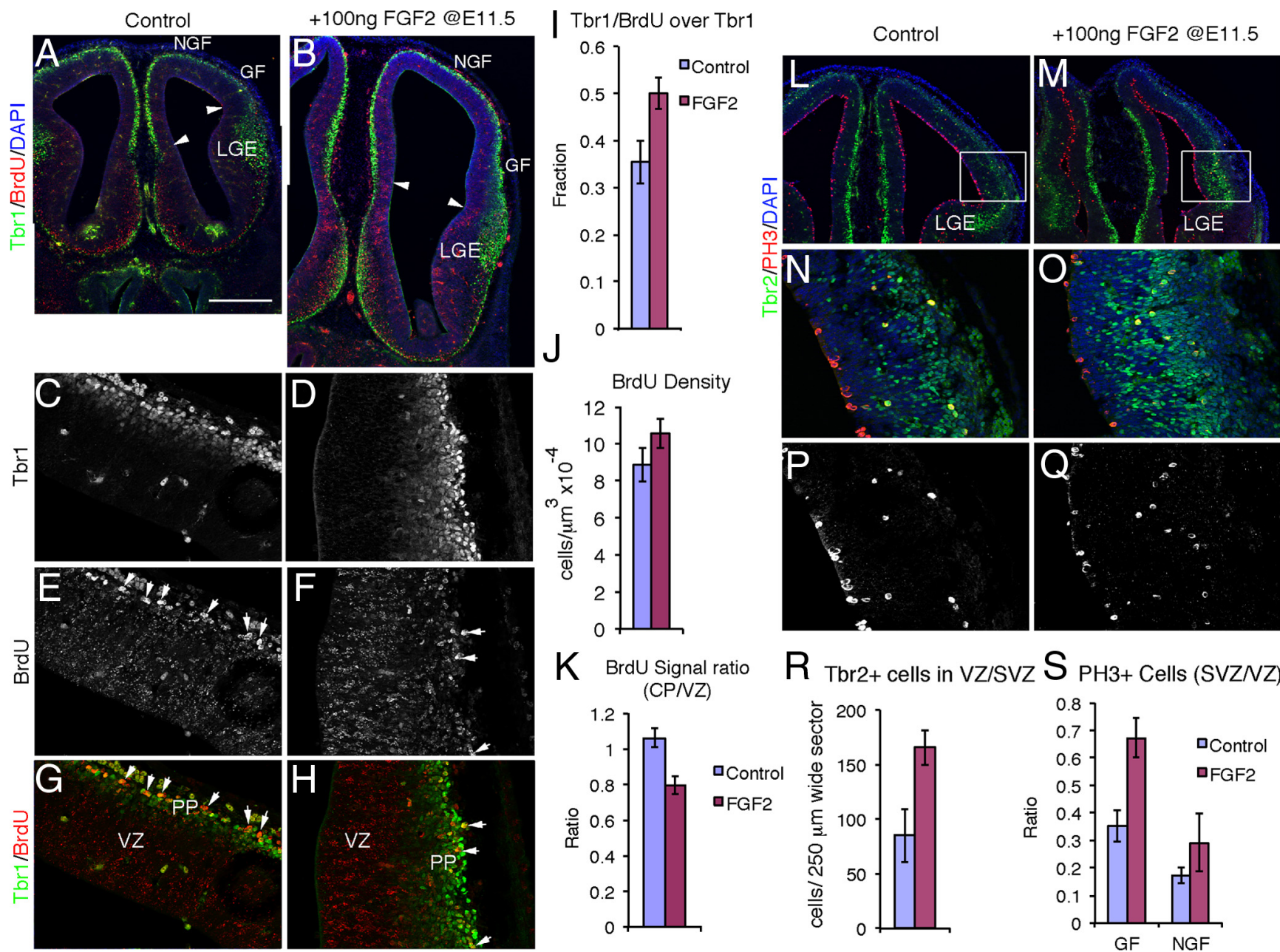

Figure 4. Decreased neurogenesis immediately after FGF2 injection followed by increased Tbr2 ${ }^{+}$intermediate progenitor proliferation and neurogenesis. BrdU was injected $4 \mathrm{~h}$ after intraventricular FGF2 injection to label cells affected by excess FGF2 activity and follow their fate. Tbr1/BrdU colabeling analysis at E13.5 $\boldsymbol{A}-\boldsymbol{H}$, indicated that more Tbr $1^{+}$cells in the cortical primordium were $\mathrm{BrdU}^{+}(\boldsymbol{I})$. However, fewer of these cells appeared to show the bright, full labeling indicative of immediate neurogenesis ( $\mathbf{C} \boldsymbol{H}$, arrows). Stereological estimates of BrdU ${ }^{+}$cell density in the cortical wall appeared to be slightly higher, but this difference was not statistically significant ( $n=3$ control embryos and $n=3$ FGF2-injected embryos; $p=0.239)(J)$. However, densitometry of the VZ versus non-VZ cortical wall showed that significantly more of the BrdU label was retained in the VZ of FGF2-injected embryos compared with controls $(n=3$ controls and $n=3 \mathrm{FGF} 2$ injected; $p=$ $0.021)(\boldsymbol{K})$. The number of Tbr2 ${ }^{+}$cells in the lateral SVZ and VZ was increased nearly twofold in FGF2-injected embryos at E13.5 $(n=3$ controls and $n=4$ FGF2 injected; $p=0.0358)(\boldsymbol{L}-\mathbf{Q}, \boldsymbol{R})$, correlating with increased appearance of PH3 ${ }^{+}$cells in the lateral SVZ $(\boldsymbol{R}, \mathbf{S})$. The ratio of SVZ/VZ PH3 ${ }^{+}$cells in the GF region; GF was greatly increased in FGF2-injected embryos $(n=3)$ compared with control embryos $(n=3)(p=0.024)$, whereas this ratio in the dorsal cortical primordium (NGF region) was not statistically different (S). PP, Preplate. Scale bar: $\mathbf{A}, \mathbf{B}, \mathbf{L}, \boldsymbol{M}, 1 \mathrm{~mm} ; \mathbf{C}-\boldsymbol{H}, \mathbf{N}-\mathbf{Q}$, $250 \mu \mathrm{m}$.

with BrdU was increased in FGF2-injected embryos, from 35.5\% in control embryos to $50.0 \%\left(\chi^{2}\right.$ value $\left.=5.24, p=0.0221\right)(n=$ 3 controls; $n=3$ FGF2-injected; Fig. $4 I$ ). Surprisingly, however, FGF2-injected embryos showed fewer brightly labeled $\mathrm{BrdU}^{+}$/ $\mathrm{Tbr}^{+}{ }^{+}$cells (Fig. $4 E-H$, arrows), although there were more that showed weaker labeling. Bright BrdU labeling is characteristic of cells that immediately became postmitotic after the period of BrdU incorporation. In contrast, cell nuclei that show only a few puncta of BrdU labeling likely divided two or more times after BrdU incorporation, diluting the labeling brightness. Overall, the BrdU density in the cortical wall was not statistically different in FGF2-injected embryos (Fig. 4J), but we observed a shift in the distribution of BrdU labeling. In FGF2-injected embryos, a greater fraction of total BrdU labeling was present in the VZ, as assessed by densitometric measurements of BrdU signal in the VZ and cortical plate in the whole hemisphere [Fig. $4 K$; the ratio of cortical plate to $\mathrm{VZ} \mathrm{BrdU}$ signal was $1.063 \pm 0.0533$ for controls $(n=3)$ and $0.796 \pm 0.048$ for FGF2-injected embryos $(n=3$; $p=0.021)]$. Together, these results suggest that, after BrdU in- corporation, there was a period of greater proliferation of stem/ progenitor cells and decreased production of $\mathrm{Tbr}^{+}$cells in embryos that received intraventricular FGF2, followed by a "catch-up" neurogenic period.

Given these results, we expected to see fewer Tbr ${ }^{+}$INP in the SVZ. However, this was not the case. We then targeted our analysis to the center of the gyrus-forming (GF) region within the rostrolateral cortical primordium (the region that shows buckling of the cortical wall) at E13.5 and counted Tbr2 ${ }^{+}$and phospho-Histone $\mathrm{H} 3$-expressing $\left(\mathrm{PH}^{+}\right)$cells. Within the GF region, we observed approximately twice the density of $\mathrm{Tbr} 2^{+}$ cells in FGF2-injected embryos compared with controls (Fig. $4 L-$ $O, R ; 85.33 \pm 24.6$ control vs $165.75 \pm 16.3$ for FGF2; $p=0.0358$ ). No such increase was found in the dorsal cortical primordium [non-gyrus-forming (NGF)] (data not shown). In addition, a greater number of mitotic $\mathrm{PH}^{+}$cells was observed in the SVZ, and the ratio of SVZ/VZ mitoses was increased in the rostrolateral neocortical wall (GF region) but not in the dorsal cortical wall (NGF region) (Fig. 4N-Q,S). These data indicate increased pro- 
duction of $\mathrm{Tbr}^{+}$progenitors, correlating with excess Er81 expression (Fig. $3 I, J)$. Hence, consistent with the idea that FGF-through Notch-antagonizes neurogenesis (Rash et al., 2011), we found that dividing progenitors throughout the rostral cortex responded in the short term after the FGF2 injection by increasing their proliferation in the VZ before eventually migrating to the cortex. Together with this generalized action, there was a concomitant local action of FGF2 in the rostrolateral neocortical wall (GF region), consisting of a sharp increase in number and proliferative activity of Tbr $2^{+}$precursors in the SVZ. Thus, in the GF region, there appeared to have been increased self-renewal of $\mathrm{RG}$ cells in the VZ as well as greater production of Tbr ${ }^{+}$INPs, either directly from RG or through increased rounds of INP self-renewal.

\section{Formation of neocortical gyri and sulci}

Next we allowed embryos injected with FGF2 at E11.5 to survive until E18.5, when the period of neurogenesis is finishing and the cortical plate can be more fully assessed. At E18.5, multiple putative gyri and sulci were often present in the rostrolateral cortical region that showed "buckling" of the cortical wall at E13.5 but not in other regions of the cortical plate (Fig. $5 A, B$ ). These structures maintained normal cortical laminar organization with respect to Tbr1, Ctip2, Zfpm2, and Cux1, except that at the bottom of an induced sulcus, the deep layers (marked by Zfpm $2^{+}$neurons) often appeared to be thinned (Fig. 5C,D). These gyri formed bilaterally in a $C d h 6^{+} ; C d h 8^{-}$parietal (somatosensory) domain of neocortex (Fig. $5 E-H$, arrows), at the border with insular cortex. To determine whether FGF2, like FGF8b, affected rostrocaudal cortical patterning, we assessed the distribution of patterning markers in sagittal sections at E18.5. Cdh6 and $C d h 8$ appeared to be expressed in appropriate locations, indicating that no significant shift in rostrocaudal patterning had occurred (Fig. 6A-E).

Remarkably, although large expansion effects were also seen in the diencephalon and midbrain as a consequence of the FGF2 injection (see below), these animals survive healthily through adulthood to an age of at least 9 months, which allowed us to examine the final effects of FGF2 on cortical gyrification. In adult mice that were injected with FGF2 at E11.5, the rostrolateral cortex was expanded macroscopically, showing a bulging region, and the animals had a raised forehead with a more rounded shape (Fig. 7A-E). Lateral views of whole adult brains showed a new, prominent gyrus in the rostrolateral cortex (Fig. $7 L, M$, asterisk).

Serial sections of two pairs of adult brains showed large fissures in a similar location to those found at E18.5, consistently occurring bilaterally in the rostrolateral neocortex (Fig. $7 F-I$, arrows), and the cortex appeared thicker in this region. More ventrally, a deepened rhinal sulcus was observed (Fig. 7G,I,O, arrowheads), and exaggerated piriform gyri were also consistently present. A short white matter radiation extended into the induced gyrus (Fig. 7G,O, blue asterisk). Sections were traced and serially reconstructed as 3D models, showing the morphology of the new sulcus (arrows) and gyrus (asterisks) in the rostrolateral neocortex (Fig. $7 P, Q$ ).

Immunohistochemistry for the upper cortical layer marker Cux1 and the neurofilament protein Smi32 demonstrated that cortical layers were intact within the induced sulci (Fig. $7 \mathrm{~J}, \mathrm{~K}$ ) and that the position of these sulci was near the neocortical/ insular cortical boundary. As at E18.5, deep layers appeared thinner at the depth of a sulcus (Fig. $7 J, K, N, O$ ). In regions unaffected by gyrification, the laminar structure of FGF2-injected animals appeared similar to controls, and neuropil labeling by Smi32 did
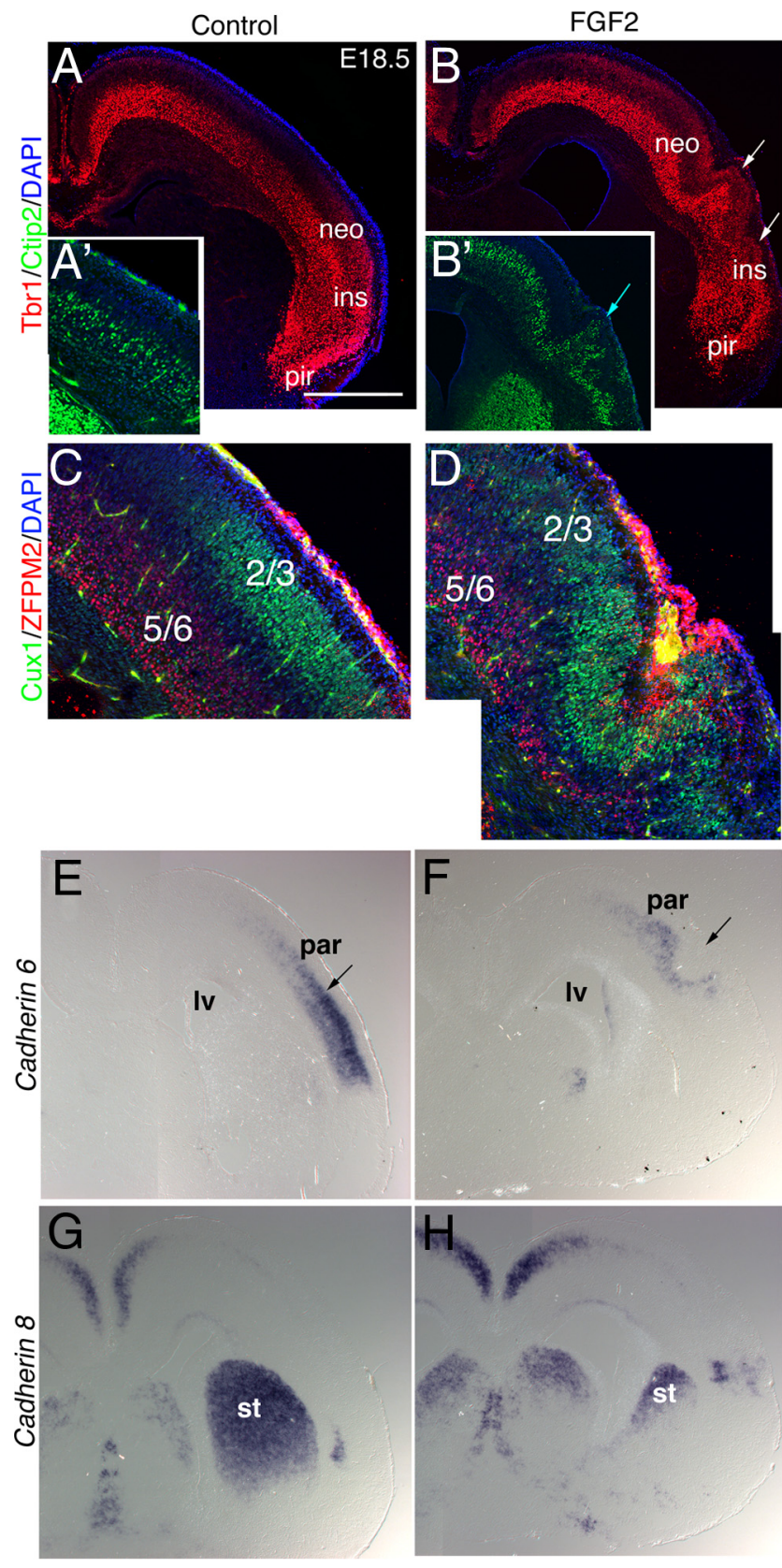

Figure 5. Induction of cortical gyri and sulci by E18.5 after FGF2 injection at E11.5. Immunohistochemistry for Tbr1/Ctip2 $\left(\boldsymbol{A}, \boldsymbol{A}^{\prime}, \boldsymbol{B}, \boldsymbol{B}^{\prime}\right)$ and Cux1/Zfpm2 (C, D), demonstrated foldings of the cortical wall at E18.5. The mouse cortical plate typically has a smooth (lissencephalic) appearance $\left(\boldsymbol{A}, \boldsymbol{A}^{\prime}, \boldsymbol{C}\right)$, but the cortical layered structure demonstrated peaks and troughs $\left(\boldsymbol{B}, \boldsymbol{B}^{\prime}, \boldsymbol{D}\right.$, arrows) consistently in both hemispheres. At the depth of induced sulci, deep layers (marked by Zfpm2, Ctip2, and Tbr1) appeared much thinner than in non-gyrified regions $\left(\boldsymbol{B}, \boldsymbol{B}^{\prime}, \boldsymbol{D}\right)$. In situ hybridization for Cdh6 and Cdh8 demonstrated that the induction of gyri and sulci (indicated by arrows) occurs in the parietal, putative somatosensory neocortex, as well as the putative insular cortex $(\boldsymbol{E}-\boldsymbol{H})$. neo, Neocortex; ins, insular cortex; Iv, Lateral ventricle; pir, piriform cortex; par, parietal cortex; st, striatum. Scale bar: $\boldsymbol{A}, \boldsymbol{B}, \boldsymbol{E}-\boldsymbol{H}, 1 \mathrm{~mm} ; \boldsymbol{C}, \boldsymbol{D}, 500 \mu \mathrm{m}$.

not reveal a defect in overall projection neuron morphology (data not shown).

The adult brains were significantly larger because of the expansion of multiple brain regions, including the diencephalon and midbrain (Fig. $7 B, C$, asterisk), and had a different overall shape. Although rostral cortex was expanded, caudal cortex and hippocampus were quite reduced in surface area (Fig. $8 A-D$ ). The dentate gyrus, CA3, and CA1 were shrunken. At E13.5, the 


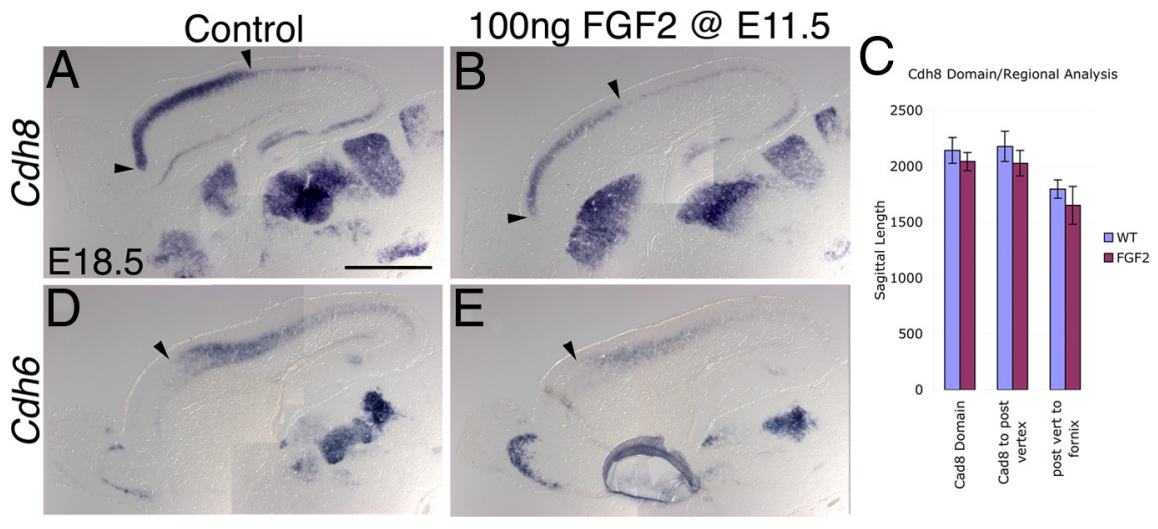

Figure 6. FGF2 injection does not alter rostrocaudal patterning of Cadherin 6/8. In situ hybridization for Cdh6 and Cdh8 at E18.5 $(\boldsymbol{A}, \boldsymbol{B}, \boldsymbol{D}, \boldsymbol{E})$. The length of the cadherin expression domain, relative to the major dimensions of the cortex, was measured in FGF2-injected embryos and compared with that of control embryos. The average length of each segment was computed and is shown in C. No significant difference was observed. Scale bar, $1 \mathrm{~mm}$.

caudal Emxl domain showed greatly reduced perimeter (Fig. $8 E, F)$. Thus, a single FGF ligand can have specific regional effects on the early growth and final size of rostral versus caudal cortex.

\section{Are axons or oRG involved in cortical gyrification?}

Together, the data presented thus far demonstrate that cortical gyri and sulci in FGF2-injected mice begin to develop before E13.5. We focused on two potential hypotheses that might explain this early folding of the neocortical wall. First, it seemed possible, although unlikely, that increased intrahemispheric axonal connections could play a role. Therefore, we examined E13.5 control and FGF2-injected brains for changes in neuropil structure and $\beta$ III tubulin ${ }^{+}$axons projecting from the nascent cortical plate. In normal mice at this age, only very short, laterally directed corticofugal axonal projections exist. We observed short corticofugal axons growing toward the future internal capsule in wildtype and FGF2-injected brains, but no hyperabundance of axons was observed in FGF2-injected brains (Fig. 9A-H), and the length of these projections appeared similar to controls. Thus, FGF2-induced gyrification is not likely to be initiated by axonal tensile effects in these mice.

We next hypothesized that oRG cells, which are defined as Pax $6^{+}$, Tbr $2^{-}$cells possessing a long basal process and lacking an apical process, might be precociously or excessively produced in the gyrifying regions of FGF2-injected brains. These cells are generally not observed in substantial numbers in normal mice and mainly at E15.5 or later (Shitamukai et al., 2011). Immunohistochemistry for Pax6, which is enriched in the dorsal telencephalon, demonstrated that the rostral cortical VZ was substantially enlarged in terms of surface area but with no major changes in thickness after FGF2 injection (Fig. 9A-F). At E13.5, the Pax6 ${ }^{+}$ VZ normally shows a sharp basal border, with extremely rare, isolated Pax6 ${ }^{+}$cells above it in the SVZ, IZ, or cortical plate (Fig. $9 A-F)$. In FGF2-injected embryos, the $\mathrm{Pax}^{+} \mathrm{VZ}$ also showed a clear basal boundary and was of similar thickness (Fig. 9D, F). In addition, the normal, elongated apical-basal polarity of Pax $6^{+}$ RG was maintained. We did not observe production of excess Pax $^{+}$cells outside of the VZ in the gyrifying region of FGF2injected embryos at E13.5 (Fig. 9A-F), suggesting that there was no substantial oRG generation outside the VZ in the gyrifying cortical primordium at this age. However, some oRGs have been reported in the outer VZ and SVZ regions (Shitamukai et al., 2011).

To determine whether oRGs might exist in greater numbers within the VZ/SVZ, we injected a GFP-expressing adenovirus together with FGF2 at E11.5 and fixed the embryos at E13.5, to allow time for the differentiation of virus-infected RG into INPs, oRGs, and neurons. All embryos that expressed adenoviral GFP $(n=5)$ were highly phenotypic in terms of incipient cortical gyrus formation, similar to the embryos shown in Figures 1 and 2. We then characterized the $\mathrm{GFP}^{+}$cells present in the VZ/SVZ of the lateral (gyrifying) versus the medial (non-gyrifying) cortical primordium, and versus those present in corresponding regions of vehicle-injected controls. Using triple immunolabeling for GFP, Pax6, and Tbr2 and blind, highresolution $3 \mathrm{D}$ imaging of cellular bodies and processes, we characterized the proportion of RGs, INPs, and oRGs derived from virally infected RGs. Because oRGs were defined $\mathrm{GFP}^{+} / \mathrm{Pax}^{+} / \mathrm{Tbr} 2^{-}$cells with a basal process but no apical process, $\mathrm{GFP}^{+}$neurons were not included in this analysis, because they would be Pax $6^{-}$and Tbr2 ${ }^{-}$. Overall, the cellular morphology of RGs, INPs, and oRGs was similar in control and FGF2-injected embryos (Fig. 9K-M). We imaged $>600 \mathrm{GFP}^{+}$progenitor cells and observed very few oRG candidates; furthermore, we did not observe significant differences in the number of putative oRGs present in any region of the cortical primordium of FGF2-injected embryos (Fig. $9 N, O)$. Thus, we do not find evidence that oRG are precociously or excessively produced during the initial gyrification of the mouse cortex.

\section{Discussion}

Here we explore the mechanisms of cortical gyrification using experimentally induced gyrification in a normally lissencephalic species. We found that gyrus formation can be induced bilaterally in stereotypical locations by increasing FGF signaling during the progenitor expansion phase of cortical development and suggest that there is neither involvement of axonal nor oRG mechanisms in the initiation of gyrus formation. We propose that a combination of increased self-renewal of RGs in the VZ and increased localized SVZ proliferative activity in the rostrolateral cortical wall results in greater surface area, as well as steeper gradient of cortical neurogenesis and localized gyrus formation.

We provide evidence that cortical surface area and overall shape can be determined by region-specific growth activities of the FGF signaling pathway, i.e., that FGFs have the capacity to expand or shrink specific cortical territories through their regulation of the stem cell pool. The role of FGFs described here is different from that described previously, in which the morphogenetic activities of FGF8 specify areal identity at a distance, acting as morphogen (Fukuchi-Shimogori and Grove, 2001; Garel et al., 2003; Toyoda et al., 2010). The additional FGF function proposed in the present paper can influence the relative size of cortical areas-but does not specify cortical areas per se-by positively or negatively regulating local surface area growth of the cortical territory in which it is active. This regulation may be in part performed through indirect effects on other signaling centers, such as the cortical hem, because our data indicate that Wnt $3 a$ in the cortical hem and Lef1 in the nearby dentate gyrus are downregulated by excess FGF signaling. The position of the major sulcus generated by FGF2 injection, encompass- 

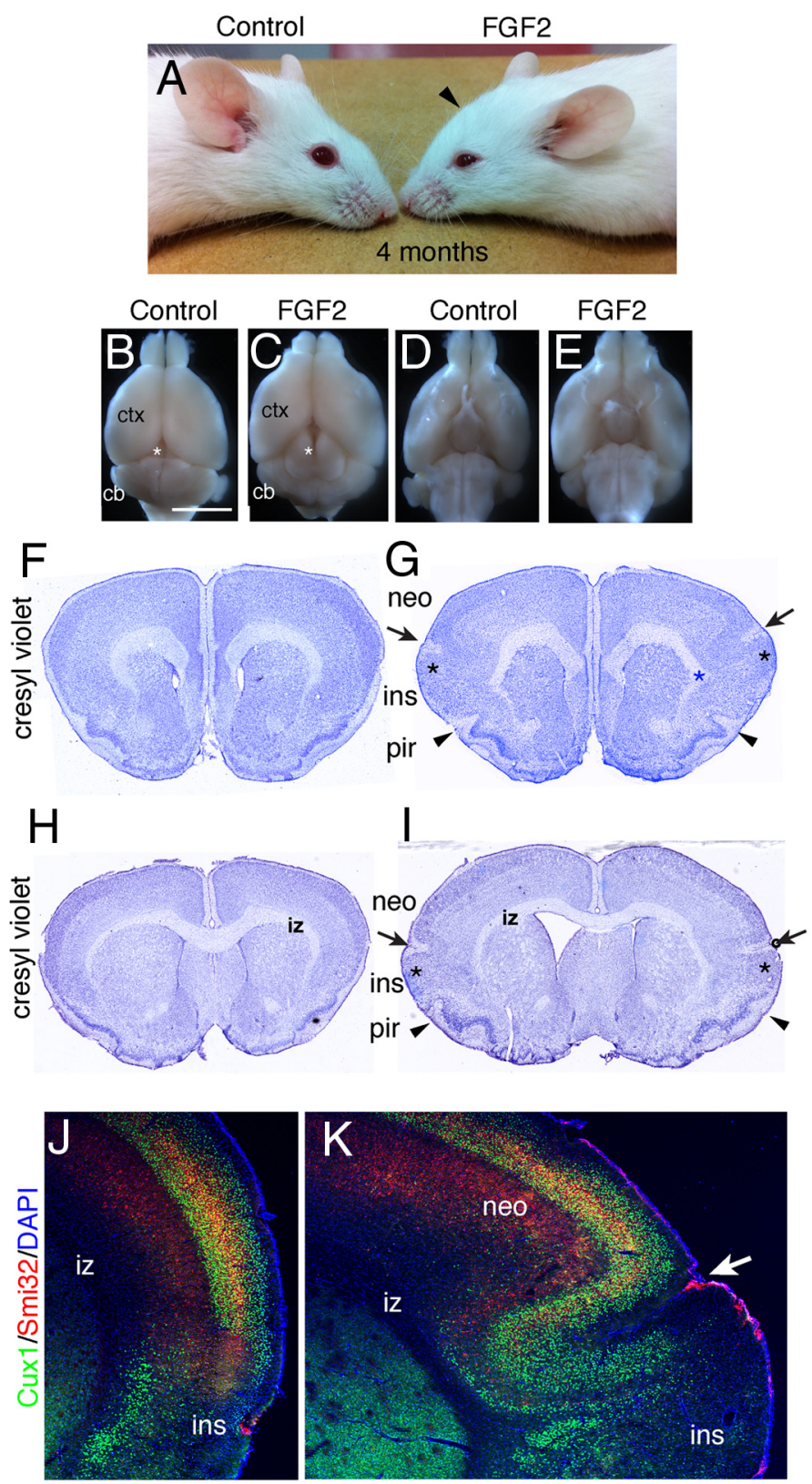
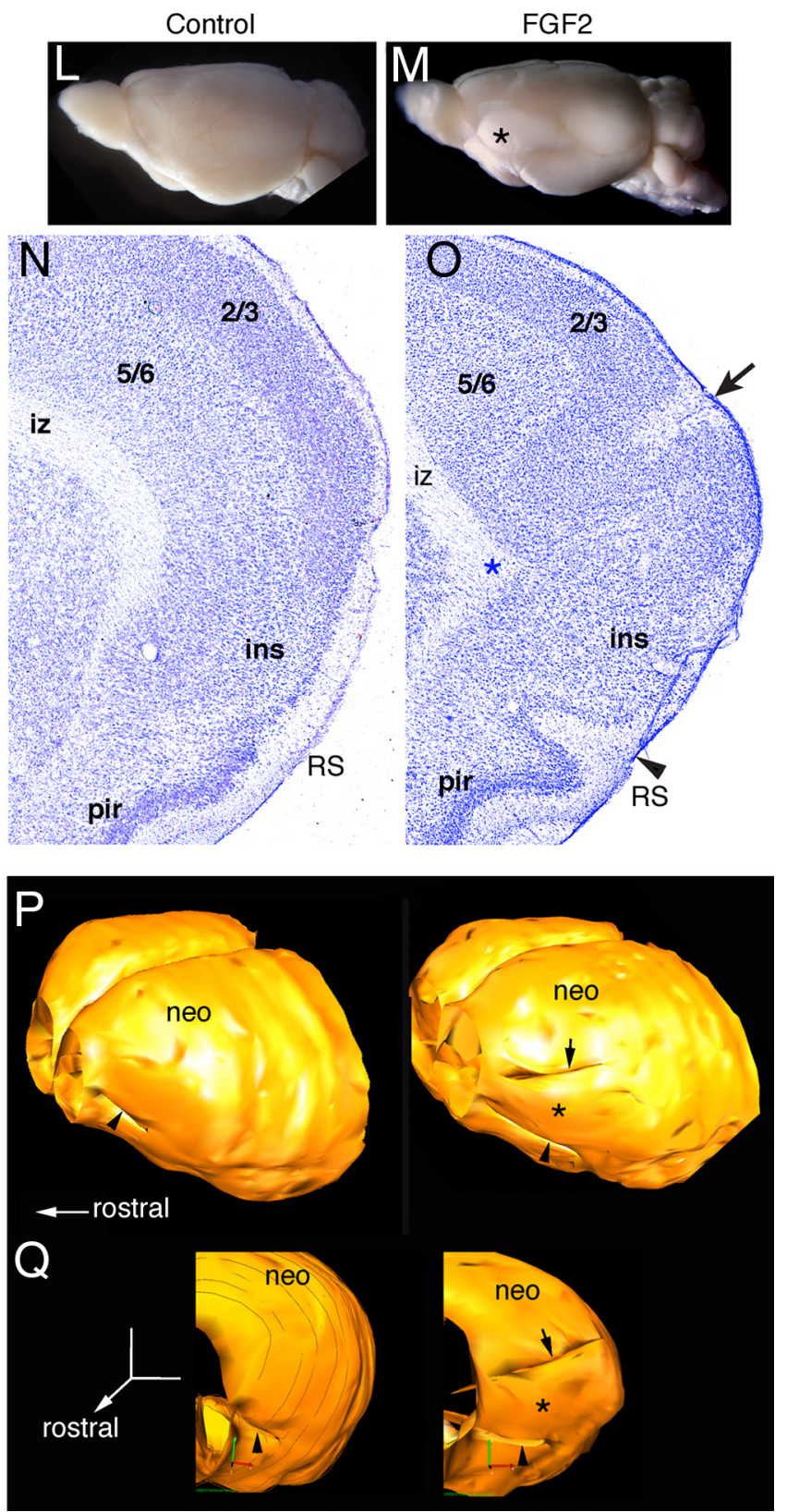

Figure 7. FGF2 injection induces fully layered cortical gyri and sulci that persist until adulthood. FGF2-injected adults showed a raised forehead ( $\boldsymbol{A}$, arrowhead). Whole-mount dorsal or ventral views of adult brains injected with FGF2 $(\boldsymbol{C}, \boldsymbol{E})$ compared with control littermates $(\boldsymbol{B}, \boldsymbol{D})$. Cresyl violet staining demonstrated deep bilateral sulci in the rostrolateral cortex in two different FGF2-injected brains ( $\boldsymbol{F}-\boldsymbol{I}$, arrows), as well as accentuated piriform gyrification ( $\mathbf{G}, \boldsymbol{I}$, arrowheads). Cortical lamination, as shown by (ux1 (green) and Smi32 (red), appeared organized around the deep fissure; neocortical layer structure ended at the sulcus depth, giving way to insular cortex $(\boldsymbol{J}, \boldsymbol{K}$, same brain shown in $\boldsymbol{H}, \boldsymbol{I})$. The lateral gyri and sulci were sometimes visible from the exterior surface of the brain ( $\boldsymbol{L}, \boldsymbol{M}$, asterisk). High-magnification views of the brain sections depicted in $\boldsymbol{F}$ and $\boldsymbol{G}$ are shown in $\boldsymbol{N}$ and $\boldsymbol{O}$. Also in this brain, cortical layers $2 / 3$ and $5 / 6$ can be seen wrapping around the deep cortical sulcus $(\mathbf{O}$, arrow), and a short white matter radiation can be seen protruding into the new gyrus from the intermediate zone $(\mathbf{G}, \mathbf{0}$, blue asterisk). $3 \mathrm{D}$ reconstructions of the surface of the brain shown in $\boldsymbol{F}, \mathbf{G}$, and $\boldsymbol{L} \mathbf{- O}$ are rendered in side views in $\boldsymbol{P}$ and rostral views in $\mathbf{Q}$, highlighting the new neocortical sulcus (arrow) and deepened rhinal sulcus (arrowhead) and gyrus (asterisk). cb, Cerebellum; iz, intermediate zone; ins, insular cortex; pir, piriform cortex; RS, Rhinal sulcus; ctx, cerebral cortex. Scale bar: $\boldsymbol{A}, 1 \mathrm{~cm} ; \boldsymbol{B}-\boldsymbol{E}, 5 \mathrm{~mm} ; \boldsymbol{F}, \boldsymbol{G}, 1.8 \mathrm{~mm} ; \boldsymbol{H}, \boldsymbol{I}, 2 \mathrm{~mm} ; \boldsymbol{J}, \boldsymbol{K}, \boldsymbol{N}, \mathbf{O}, 350 \mu \mathrm{m} ; \boldsymbol{L}, \boldsymbol{M}$, $3 \mathrm{~mm} ; \boldsymbol{P}, \mathbf{Q}, 1.5 \mathrm{~mm}$.

ing extreme lateral neocortex and the dorsal border of insular cortex, is the site of the deepest (and first to form) fissure in the carnivore and primate brain - the sylvian fissure. A separate (and deeper) rhinal fissure was also observed. This gyral positioning appears to be molecularly specified, in accordance with our data indicating a regionally specific increase in Er81 expression and $\mathrm{Tbr} 2{ }^{+}$cell proliferation in the rostrolateral neocortical primordium.

\section{Mechanisms of gyrus formation}

Previous studies showed production of either an expanded cortical surface or additional structures similar to cortical gyri and sulci in the mouse brain. This has been done by increasing proliferation in the cortical VZ using a constitutively active $\beta$-catenin (Chenn and Walsh, 2002) or by decreasing cell death by deletion of Caspase 9 (Kuida et al., 1998) or EphrinA7 mutagenesis (Depaepe et al., 2005; for review, see Haydar et al., 


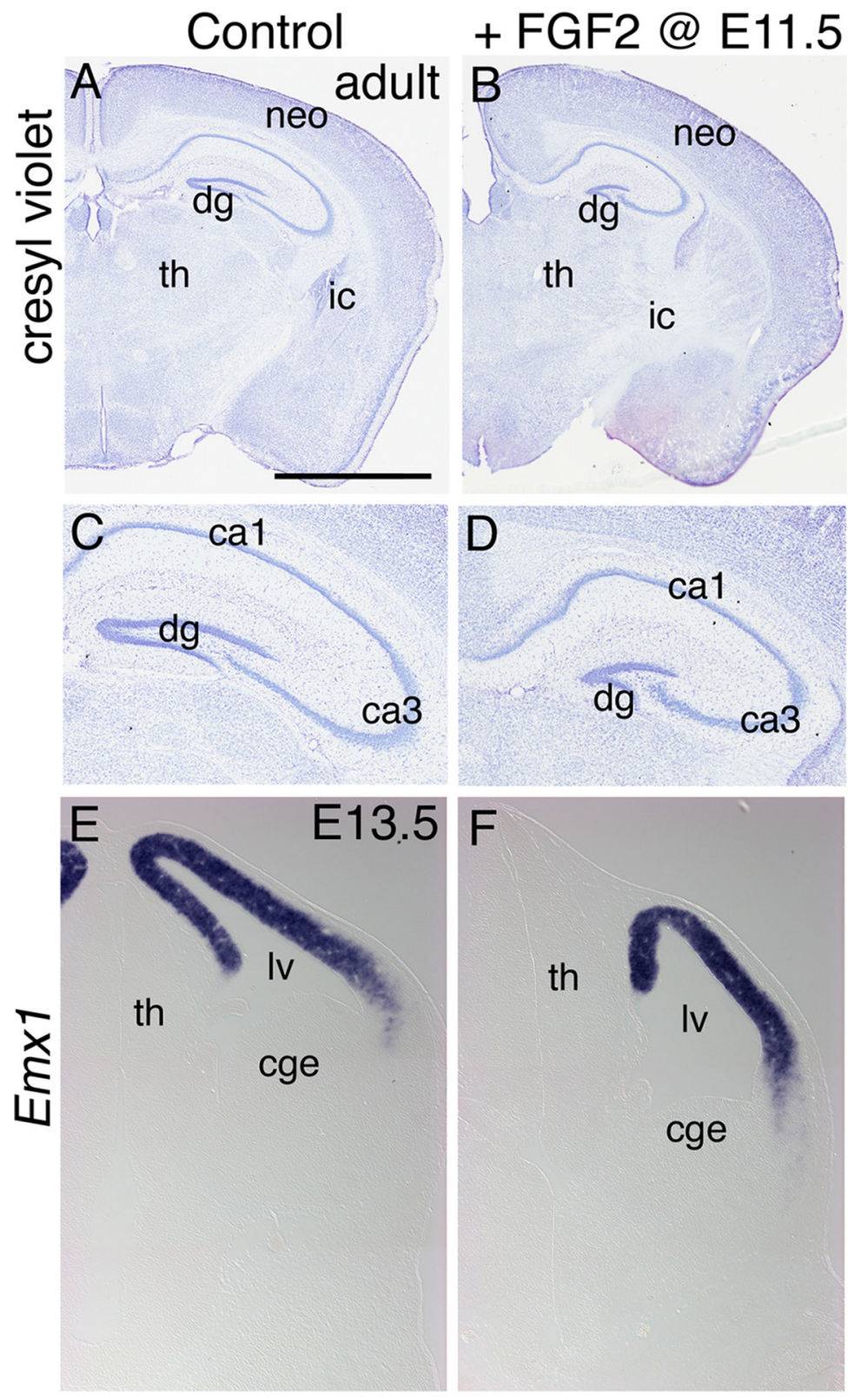

Figure 8. Impaired hippocampal growth in FGF2-injected mice. Although the rostral cortex was enlarged, the hippocampus was severely reduced in size in adult FGF2-injected brains, as shown by cresyl violet staining $(\boldsymbol{A}-\boldsymbol{D})$. The brains shown in $\boldsymbol{A}-\boldsymbol{D}$ are the same as in Figure $8, \boldsymbol{A}-\boldsymbol{E}$ and $\boldsymbol{H}-\boldsymbol{K}$. The CA1, CA3, and dentate gyrus regions were clearly identifiable in FGF2-injected brains, indicating a high level of organization $(\boldsymbol{C}, \boldsymbol{D})$. In addition, the growth of the hippocampal primordium, labeled by $E m \times 1$, was drastically curtailed just $48 \mathrm{~h}$ after FGF2 injection at E11.5 $(\boldsymbol{E}, \boldsymbol{F})$. dg, Dentate gyrus; neo, neocortex; ic, internal capsule; th, thalamus; Iv, lateral ventricle; cge, caudal ganglionic eminence. Scale bar: $A, B, 2 \mathrm{~mm} ; C-F, 1 \mathrm{~mm}$.

1999). More recently, retinoic acid secreted from the meninges was shown to promote cortical neurogenesis, regulating the size of the VZ progenitor pool and its surface area (Siegenthaler et al., 2009). However, the resultant brains generally appeared quite disorganized and showed major cortical heterotopias and malformations. Furthermore, these previous models of gyrified brains did not produce viable adult offspring (Chenn and Walsh, 2002; Hatakeyama et al., 2004). In contrast, our mouse model is the first model of well-formed, fully layered neocortical gyri/sulci that survives healthily until adulthood.

Results described previously indicate that gyrification is not induced by FGF2 injections at E15.5 in the rat (Vaccarino et al., 1999). Crucially, this time point corresponds to E13.5-E14.0 in mouse, which is after the progenitor expansion phase has ended and during the neurogenesis of cortical layers 5 and 4 (Takahashi et al., 1999). This suggests that the cortical primordium is able to initiate gyrification during the RG expansion phase, which normally ends before E13.5 in CD-1 mice (Rash et al., 2011) but, apparently, not after the expansion phase is complete. Gyrification occurs before the formation of intrahemispheric axonal connections, indicating that folding of the cortical wall underlying gyrus formation was independent of axonal tensile effects.

The raised forehead of adult FGF2injected mice described here demonstrates that skull volume and shape are sensitive to alterations in cortical expansion before the period of chondrogenesis and ossification. Because we did not find evidence supporting the involvement of axonal, ossification, or oRG mechanisms in cortical gyrification, we refocused on the VZ expansion and excessive INP activity we observed at E13.5.

Only in the dorsolateral cortical wall, near the boundary of neocortex and basal ganglia, was gyrification observed, in apparent spatial correspondence with localized overproduction of INPs in the SVZ and ectopic expression of Er81, a known downstream FGF gene. It may be that the ratio of SVZ neurogenesis/self-renewal to $\mathrm{VZ}$ neurogenesis/self-renewal is responsible for gyrification. Indeed, studies indicate that primates show multiple rounds of $\mathrm{Tbr} 2^{+}$INP divisions in the SVZ and outer SVZ, whereas the INPs of rodents typically divide only once (Kriegstein et al., 2006). Our data support this view, because adenovirally labeled $\mathrm{RGs}$ did not generate an excess of INPs (Fig. 9N,O), but yet we observed greater numbers of them (Fig. $4 L-$ $S)$. This suggests that the excess INPs did not originate from the VZ. We suggest that the sharpness of such regional effects, i.e., the rate of change of this ratio along the cortical wall, may produce an initial buckling of the pallial surface that is magnified through time, i.e., further compressed into tighter cortical folds by the expanding cortical tissue itself.

In this respect, although we think that axonal mechanisms are unlikely to be involved in gyrus initiation, we do not rule out axonal mechanisms in the later development of cortical gyri. Indeed, in utero experimental modification of axonal input to the visual cortex has been shown to affect gyrification patterns of monkey visual cortex (Goldman-Rakic and Rakic, 1984). Similarly, we do not exclude a later role for oRGs, but in FGF2-injected embryos, oRGs were not found in a spatiotemporal location to be able to initiate cortical gyrification by E13.5. Analysis of lissencephalic primates and gyrencephalic rodents also does not appear to confirm a necessary role for 
oRGs in gyrus formation (GarcíaMoreno et al., 2012; Kelava et al., 2012; for review, see Hevner and Haydar, 2012). It also remains possible that skull and meningeal development may contribute to the morphogenesis of cortical gyri as in the ferret (Smart and McSherry, 1986).

In FGF2-injected embryos, the discordance between VZ and SVZ activity (i.e., the increased self-renewal of RG as well as the excess Tbr2 ${ }^{+}$cells) is most easily explained by the temporary effect of FGF2 promoting self-renewal of both classes of progenitors. In this regard, SVZ progenitors are known to temporarily move down to the $\mathrm{VZ}$ and send projections that may reach the ventricle (Noctor et al., 2004). Because SVZ progenitors bear Notch ligands and are themselves responsive to Notch signaling (Mizutani et al., 2007), this would have the effect of simultaneously increasing proliferation of RG in the VZ and INPs in the SVZ, as we observed. Thus, expanding the thickness of the SVZ and preplate of the cortical wall focally, relative to a tangential expansion of VZ that is unaltered in thickness, may be at the root of cortical folding.

On the diversity of FGF ligand-receptor interactions and regional specificity of action

Multiple FGF ligands and receptors are expressed in very different spatiotemporal patterns. It is now becoming evident that different FGF ligands have opposite effects on cortical growth (Borello et al., 2008), although the sum of all FGF effects is to promote surface area growth both rostrally and caudally through the selfrenewal of cortical RGs (Rash et al., 2011). We now show that a single FGF ligand (FGF2) can have opposite growth effects on different cortical regions, likely attributable in part to indirect effects on the expression of signals such as Wnt $3 a$ within the cortical hem. This additional complexity provides a conceptual basis for selective expansion (and even shrinkage) of cortical regions during evolution. Thus, future studies of cortical patterning should take into account the localized growth effects of patterning genes. We speculate that higher mammals will show substantial differences in the spatiotemporal expression patterns of various FGF ligands and receptors, or variations in their activities. In higher mammals, the radial progenitor expansion phase is much longer (Kriegstein et al., 2006; Dehay and Kennedy, 2007; Rakic, 2009), producing larger cortical primordia, which may respond differently to focal sources of FGF activities when distances from

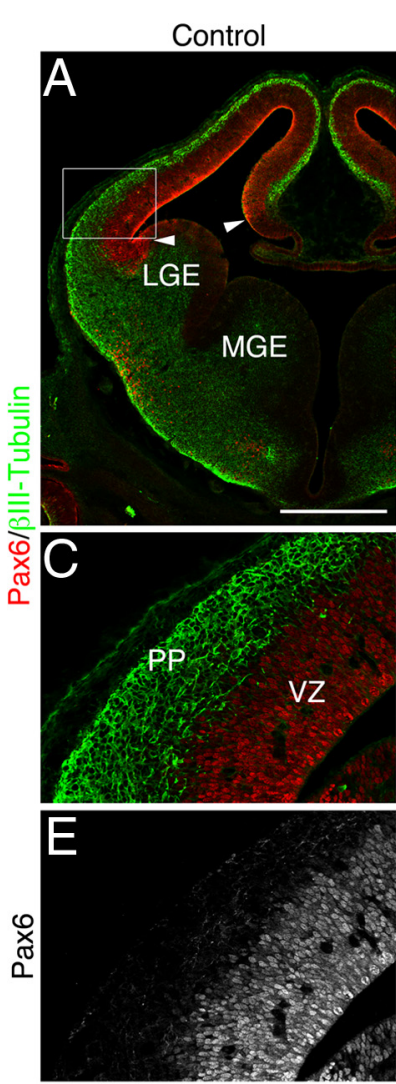

+100ng FGF2 @ E11.5
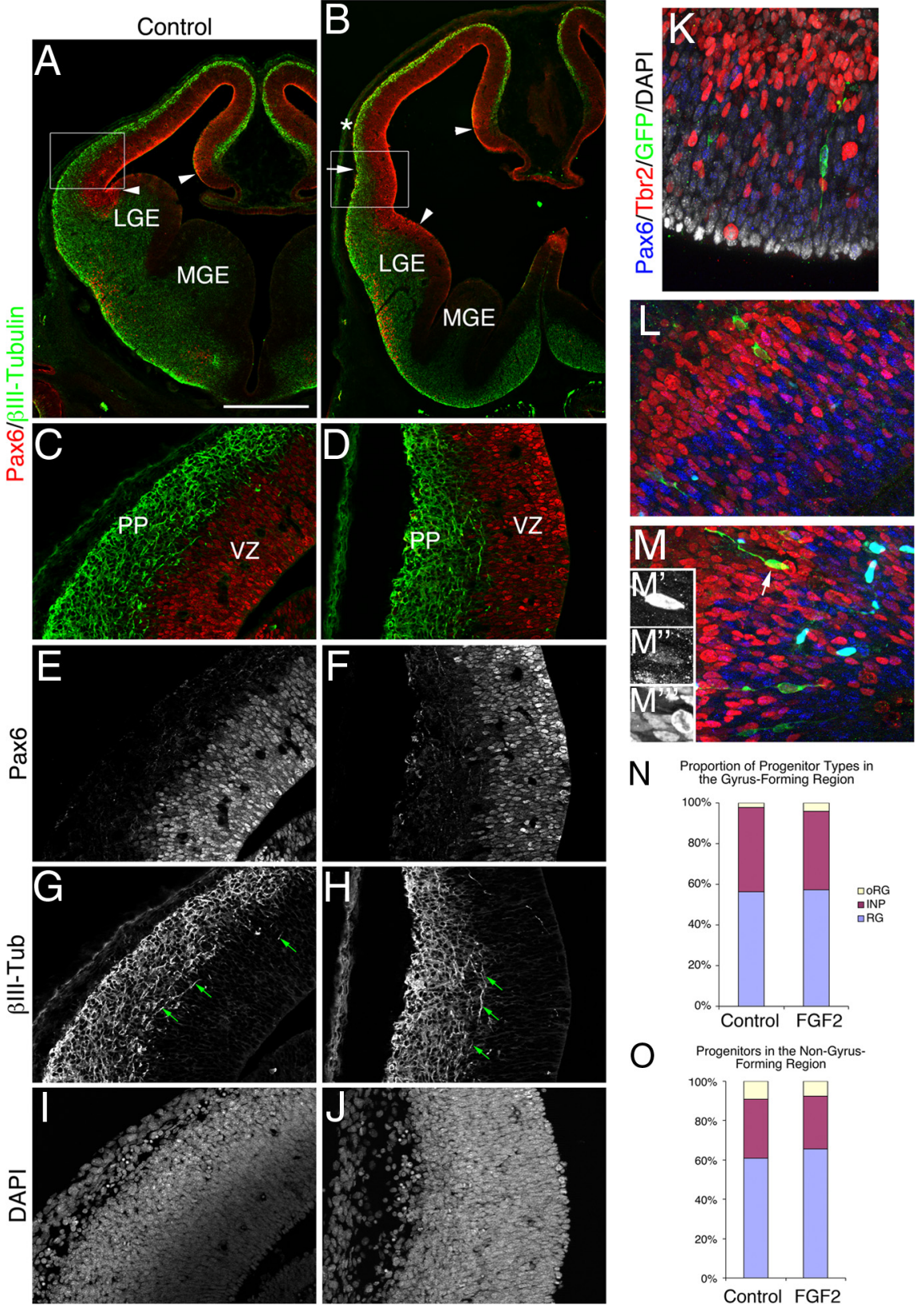

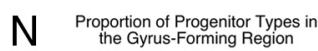
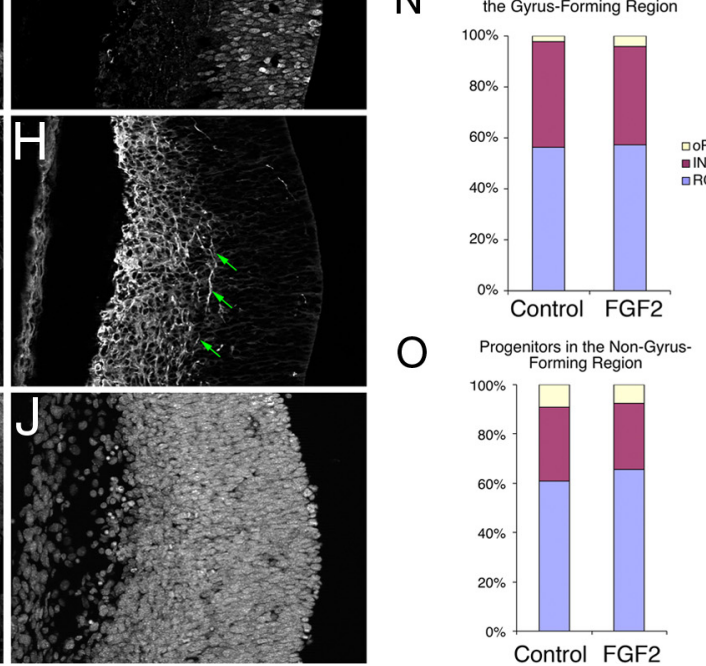

O

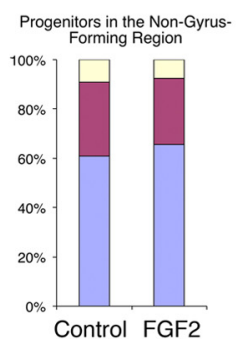

Figure 9. oRG and axonal connections do not contribute significantly to initial stages of gyrus formation after FGF2 injection. Staining for Pax6 and $\beta$ III tubulin $(\boldsymbol{A}-\boldsymbol{J})$ showed an orderly VZ in both control and FGF2-injected embryos at E13.5. $(-\mathcal{J}$ are high-magnification views of the boxed regions in $\boldsymbol{A}$ and $\boldsymbol{B}$. Very rare Pax ${ }^{+}$cells were observed outside of the VZ proper in both control and FGF2-injected embryos $(\boldsymbol{A}-\boldsymbol{F})$. However, there was no apparent increase of these cells in the GF lateral cortical primordium versus NGF regions. Axons and neuropil stained for $\beta$ III tubulin showed the presence of very few laterally projecting corticofugal axons, with no hyperabundance in the gyrifying region $(A, B, G, H$, green arrows). $I, J, D A P I$ counterstain. Quantification of adenoviral GFP labeling of $>600$ cortical progenitors at the time of FGF2 injection at E11.5 $\boldsymbol{K}-\mathbf{O}$ revealed no significant difference in the proportions of $\mathrm{RG}\left(\boldsymbol{K} ; \mathrm{Pax}^{+} / \mathrm{GFP}^{+}\right)$, intermediate neuronal precursors $\left(\boldsymbol{L} ; \mathrm{Tbr2}{ }^{+} / \mathrm{GFP}{ }^{+}\right)$, or oRG $\left(\boldsymbol{M} ; \mathrm{Tbr2}{ }^{-} /\right.$ $\mathrm{Pax}^{+} / \mathrm{GFP}^{+}$cells in the outer VZ or SVZ lacking an apical process, arrow) in both gyrifying $(\boldsymbol{N} ; n=3$ controls and $n=4$ FGF2-injected brains; $p=0.90, p=0.81$, and $p=0.53$ for RGs, INPs, and oRGs, respectively) and non-gyrifying ( $\mathbf{0} ; p=0.63, p=$ 0.72 , and $p=0.79$ for RGs, INPs, and oRGs, respectively) regions. Single-channel GFP, Pax6, and Tbr2 $z$-stack images for the oRG cell in $\boldsymbol{M}$ (arrow) are shown in $\boldsymbol{M}^{\prime}, \boldsymbol{M}^{\prime \prime}$, and $\boldsymbol{M}^{\prime \prime \prime}$, respectively. MGE, Medial ganglionic eminence; PP, preplate. Scale bar: $A, B, 1 \mathrm{~mm}$; (-J, $250 \mu \mathrm{m} ; \boldsymbol{K}-\mathbf{M}, 125 \mu \mathrm{m}$.

ligand source to target cell increase. Higher mammals may thus be afforded more opportunities for more (and deeper) cortical folds. The increased length of time in which primate gyri form during gestation allows the possibility that other mechanisms come into play. For example, axonal tension and oRGs may be 
much more of a factor in these species than in the mouse, as shown here.

It remains unclear which receptors are responsible for the differential activities of FGF2. Future work should examine the effects of additional FGF ligand injections in FGFR1, FGFR2, or FGFR3 knock-out embryos to ascertain the specificity of ligand-receptor interactions. Furthermore, different combinations of FGF ligands may produce unique effects on the cortical primordium, and the relative dosages of each ligand would be expected to determine the growth outcome.

It was remarkable that large changes in brain structure did not appear to affect the cortical layer structure of FGF2-injected animals. Indeed, both the disruption (Rash et al., 2011) and the hyperactivation of the FGF system altered the dynamics of cortical surface area expansion but did not appreciably disturb cortical layer structure. Thus, the overall activities of at least two different FGF ligands can be substantially modulated without major negative consequences. Such a system would seem to be ideal for exploitation during the course of the evolution of cognitive functions typical of gyrencephalic species.

\section{References}

Borello U, Cobos I, Long JE, McWhirter JR, Murre C, Rubenstein JL (2008) FGF15 promotes neurogenesis and opposes FGF8 function during neocortical development. Neural Dev 3:17. CrossRef Medline

Chellaiah A, Yuan W, Chellaiah M, Ornitz DM (1999) Mapping ligand binding domains in chimeric fibroblast growth factor receptor molecules. Multiple regions determine ligand binding specificity. J Biol Chem 274: 34785-34794. CrossRef Medline

Chenn A, Walsh CA (2002) Regulation of cerebral cortical size by control of cell cycle exit in neural precursors. Science 297:365-369. CrossRef Medline

Cholfin JA, Rubenstein JL (2007) Patterning of frontal cortex subdivisions by Fgf17. Proc Natl Acad Sci U S A 104:7652-7657. CrossRef Medline

Dehay C, Kennedy H (2007) Cell-cycle control and cortical development. Nat Rev Neurosci 8:438-450. CrossRef Medline

Depaepe V, Suarez-Gonzalez N, Dufour A, Passante L, Gorski JA, Jones KR, Ledent C, Vanderhaeghen P (2005) Ephrin signalling controls brain size by regulating apoptosis of neural progenitors. Nature 435:1244-1250. CrossRef Medline

Fukuchi-Shimogori T, Grove EA (2001) Neocortex patterning by the secreted signaling molecule FGF8. Science 294:1071-1074. CrossRef Medline

García-Moreno F, Vasistha NA, Trevia N, Bourne JA, Molnár Z (2012) Compartmentalization of cerebral cortical germinal zones in a lissencephalic primate and gyrencephalic rodent. Cereb Cortex 22:482-492. CrossRef Medline

Garel S, Huffman KJ, Rubenstein JL (2003) Molecular regionalization of the neocortex is disrupted in Fgf8 hypomorphic mutants. Development 130: 1903-1914. CrossRef Medline

Goldman-Rakic P, Rakic P (1984) Experimental modification of gyral patterns. In: Cerebral dominance: the biological foundations (Geschwind N, Galaburda AM, eds). Cambridge, MA: Harvard UP.

Grove EA, Tole S, Limon J, Yip L, Ragsdale CW (1998) The hem of the embryonic cerebral cortex is defined by the expression of multiple Wnt genes and is compromised in Gli-3 deficient mice. Development 125: 2315-2325. Medline

Hatakeyama J, Bessho Y, Katoh K, Ookawara S, Fujioka M, Guillemot F, Kageyama R (2004) Hes genes regulate size, shape and histogenesis of the nervous system by control of the timing of neural stem cell differentiation. Development 131:5539-5550. CrossRef Medline

Haydar TF, Kuan CY, Flavell RA, Rakic P (1999) The role of cell death in regulating the size and shape of the mammalian forebrain. Cereb Cortex 9:621-626. CrossRef Medline

Hébert JM, Fishell G (2008) The genetics of early telencephalon patterning: some assembly required. Nat Rev Neurosci 9:678-685. CrossRef Medline

Hevner RF, Haydar TF (2012) The (not necessarily) convoluted role of basal radial glia in cortical neurogenesis. Cereb Cortex 22:465-468. CrossRef Medline
Kelava I, Reillo I, Murayama AY, Kalinka AT, Stenzel D, Tomancak P, Matsuzaki F, Lebrand C, Sasaki E, Schwamborn JC, Okano H, Huttner WB, Borrell V (2012) Abundant occurrence of basal radial glia in the subventricular zone of embryonic neocortex of a lissencephalic primate, the common marmoset Callithrix jacchus. Cereb Cortex 22:469-481. CrossRef Medline

Kriegstein A, Noctor S, Martínez-Cerdeño V (2006) Patterns of neural stem and progenitor cell division may underlie evolutionary cortical expansion. Nat Rev Neurosci 7:883-890. CrossRef Medline

Kuida K, Haydar TF, Kuan CY, Gu Y, Taya C, Karasuyama H, Su MS, Rakic P, Flavell RA (1998) Reduced apoptosis and cytochrome c-mediated caspase activation in mice lacking caspase 9. Cell 94:325-337. CrossRef Medline

Lee SM, Tole S, Grove E, McMahon AP (2000) A local Wnt-3a signal is required for development of the mammalian hippocampus. Development 127:457-467. Medline

Lui JH, Hansen DV, Kriegstein AR (2011) Development and evolution of the human neocortex. Cell 146:18-36. CrossRef Medline

Malatesta P, Hartfuss E, Götz M (2000) Isolation of radial glial cells by fluorescent-activated cell sorting reveals a neuronal lineage. Development 127:5253-5263. Medline

Mizutani K, Yoon K, Dang L, Tokunaga A, Gaiano N (2007) Differential Notch signalling distinguishes neural stem cells from intermediate progenitors. Nature 449:351-355. CrossRef Medline

Nie J, Guo L, Li K, Wang Y, Chen G, Li L, Chen H, Deng F, Jiang X, Zhang T, Huang L, Faraco C, Zhang D, Guo C, Yap PT, Hu X, Li G, Lv J, Yuan Y, Zhu D, et al. (2012) Axonal fiber terminations concentrate on gyri. Cereb Cortex 22:2831-2839. CrossRef Medline

Noctor SC, Martínez-Cerdeño V, Ivic L, Kriegstein AR (2004) Cortical neurons arise in symmetric and asymmetric division zones and migrate through specific phases. Nat Neurosci 7:136-144. CrossRef Medline

Ohtsuka T, Sakamoto M, Guillemot F, Kageyama R (2001) Roles of the basic helix-loop-helix genes Hes1 and Hes5 in expansion of neural stem cells of the developing brain. J Biol Chem 276:30467-30474. CrossRef Medline

Raballo R, Rhee J, Lyn-Cook R, Leckman JF, Schwartz ML, Vaccarino FM (2000) Basic fibroblast growth factor (Fgf2) is necessary for cell proliferation and neurogenesis in the developing cerebral cortex. J Neurosci 20: 5012-5023. Medline

Rakic P (1988) Specification of cerebral cortical areas. Science 241:170-176. CrossRef Medline

Rakic P (1995) A small step for the cell, a giant leap for mankind: a hypothesis of neocortical expansion during evolution. Trends Neurosci 18:383388. CrossRef Medline

Rakic P (2009) Evolution of the neocortex: a perspective from developmental biology. Nat Rev Neurosci 10:724-735. CrossRef Medline

Rash BG, Grove EA (2006) Area and layer patterning in the developing cerebral cortex. Curr Opin Neurobiol 16:25-34. CrossRef Medline

Rash BG, Grove EA (2007) Patterning the dorsal telencephalon: a role for sonic hedgehog? J Neurosci 27:11595-11603. CrossRef Medline

Rash BG, Lim HD, Breunig JJ, Vaccarino FM (2011) FGF signaling expands embryonic cortical surface area by regulating Notch-dependent neurogenesis. J Neurosci 31:15604-15617. CrossRef Medline

Reillo I, de Juan Romero C, García-Cabezas MÁ, Borrell V (2011) A role for intermediate radial glia in the tangential expansion of the mammalian cerebral cortex. Cereb Cortex 21:1674-1694. CrossRef Medline

Sahara S, O'Leary DD (2009) Fgf10 regulates transition period of cortical stem cell differentiation to radial glia controlling generation of neurons and basal progenitors. Neuron 63:48-62. CrossRef Medline

Seuntjens E, Nityanandam A, Miquelajauregui A, Debruyn J, Stryjewska A, Goebbels S, Nave KA, Huylebroeck D, Tarabykin V (2009) Sip1 regulates sequential fate decisions by feedback signaling from postmitotic neurons to progenitors. Nat Neurosci 12:1373-1380. CrossRef Medline

Shitamukai A, Konno D, Matsuzaki F (2011) Oblique radial glial divisions in the developing mouse neocortex induce self-renewing progenitors outside the germinal zone that resemble primate outer subventricular zone progenitors. J Neurosci 31:3683-3695. CrossRef Medline

Siegenthaler JA, Ashique AM, Zarbalis K, Patterson KP, Hecht JH, Kane MA, Folias AE, Choe Y, May SR, Kume T, Napoli JL, Peterson AS, Pleasure SJ (2009) Retinoic acid from the meninges regulates cortical neuron generation. Cell 139:597-609. CrossRef Medline

Smart IH, McSherry GM (1986) Gyrus formation in the cerebral cortex of 
the ferret. II. Description of the internal histological changes. J Anat 147: 27-43. Medline

Smith KM, Ohkubo Y, Maragnoli ME, Rasin MR, Schwartz ML, Sestan N, Vaccarino FM (2006) Midline radial glia translocation and corpus callosum formation require FGF signaling. Nat Neurosci 9:787-797. CrossRef Medline

Stevens HE, Smith KM, Maragnoli ME, Fagel D, Borok E, Shanabrough M, Horvath TL, Vaccarino FM (2010) Fgfr2 is required for the development of the medial prefrontal cortex and its connections with limbic circuits. J Neurosci 30:5590-5602. CrossRef Medline

Storm EE, Garel S, Borello U, Hebert JM, Martinez S, McConnell SK, Martin GR, Rubenstein JL (2006) Dose-dependent functions of Fgf8 in regulating telencephalic patterning centers. Development 133: 1831-1844. CrossRef Medline

Takahashi T, Nowakowski RS, Caviness VS Jr (1996) The leaving or Q fraction of the murine cerebral proliferative epithelium: a general model of neocortical neuronogenesis. J Neurosci 16:6183-6196. Medline

Takahashi T, Goto T, Miyama S, Nowakowski RS, Caviness VS, Jr (1999)
Sequence of neuron origin and neocortical laminar fate: relation to cell cycle of origin in the developing murine cerebral wall. J Neurosci 19: 10357-10371. Medline

Toyoda R, Assimacopoulos S, Wilcoxon J, Taylor A, Feldman P, SuzukiHirano A, Shimogori T, Grove EA (2010) FGF8 acts as a classic diffusible morphogen to pattern the neocortex. Development 137: 3439-3448. CrossRef Medline

Vaccarino FM, Schwartz ML, Raballo R, Nilsen J, Rhee J, Zhou M, Doetschman T, Coffin JD, Wyland JJ, Hung YT (1999) Changes in cerebral cortex size are governed by fibroblast growth factor during embryogenesis. Nat Neurosci 2:246-253. CrossRef Medline

Van Essen DC (1997) A tension-based theory of morphogenesis and compact wiring in the central nervous system. Nature 385:313-318. CrossRef Medline

Wang X, Tsai JW, LaMonica B, Kriegstein AR (2011) A new subtype of progenitor cell in the mouse embryonic neocortex. Nat Neurosci 14:555561. CrossRef Medline 